\title{
Whole-transcriptome, high-throughput RNA sequence analysis of the bovine macrophage response to Mycobacterium bovis infection in vitro
}

Nicolas C Nalpas ${ }^{1}$, Stephen DE Park ${ }^{1}$, David A Magee ${ }^{1}$, Maria Taraktsoglou', John A Browne ${ }^{1}$, Kevin M Conlon², Kévin Rue-Albrecht ${ }^{1}$, Kate E Killick ${ }^{1}$, Karsten Hokamp ${ }^{3}$, Amanda J Lohan ${ }^{4}$, Brendan J Loftus ${ }^{4}$, Eamonn Gormley ${ }^{5}$, Stephen V Gordon ${ }^{2,4}$ and David E MacHugh ${ }^{1,4^{*}}$

\begin{abstract}
Background: Mycobacterium bovis, the causative agent of bovine tuberculosis, is an intracellular pathogen that can persist inside host macrophages during infection via a diverse range of mechanisms that subvert the host immune response. In the current study, we have analysed and compared the transcriptomes of M. bovis-infected monocytederived macrophages (MDM) purified from six Holstein-Friesian females with the transcriptomes of non-infected control MDM from the same animals over a $24 \mathrm{~h}$ period using strand-specific RNA sequencing (RNA-seq). In addition, we compare gene expression profiles generated using RNA-seq with those previously generated by us using the high-density Affymetrix ${ }^{\circledR}$ GeneChip ${ }^{\circledR}$ Bovine Genome Array platform from the same MDM-extracted RNA.

Results: A mean of 7.2 million reads from each MDM sample mapped uniquely and unambiguously to single Bos taurus reference genome locations. Analysis of these mapped reads showed 2,584 genes (1,392 upregulated; 1,192 downregulated) and 757 putative natural antisense transcripts (558 upregulated; 119 downregulated) that were differentially expressed based on sense and antisense strand data, respectively (adjusted $P$-value $\leq 0.05$ ). Of the differentially expressed genes, 694 were common to both the sense and antisense data sets, with the direction of expression (i.e. up- or downregulation) positively correlated for 693 genes and negatively correlated for the remaining gene. Gene ontology analysis of the differentially expressed genes revealed an enrichment of immune, apoptotic and cell signalling genes. Notably, the number of differentially expressed genes identified from RNA-seq sense strand analysis was greater than the number of differentially expressed genes detected from microarray analysis (2,584 genes versus 2,015 genes). Furthermore, our data reveal a greater dynamic range in the detection and quantification of gene transcripts for RNA-seq compared to microarray technology.

Conclusions: This study highlights the value of RNA-seq in identifying novel immunomodulatory mechanisms that underlie host-mycobacterial pathogen interactions during infection, including possible complex post-transcriptional regulation of host gene expression involving antisense RNA.
\end{abstract}

Keywords: Bovine tuberculosis, Immune response, Microarray, Mycobacterium bovis, Natural antisense transcript, RNA-sequencing, Transcriptome

\footnotetext{
* Correspondence: david.machugh@ucd.ie

'Animal Genomics Laboratory, UCD School of Agriculture and Food Science,

University College Dublin, Belfield, Dublin 4, Dublin, Ireland

${ }^{4} U C D$ Conway Institute of Biomolecular and Biomedical Research, University

College Dublin, Belfield, Dublin 4, Dublin, Ireland

Full list of author information is available at the end of the article
} 


\section{Background}

Bovine tuberculosis (BTB) is caused by infection with Mycobacterium bovis-an intracellular pathogen belonging to the Mycobacterium tuberculosis complex [1-4]. BTB has major economic, animal welfare and public health consequences, and has remained recalcitrant to eradication despite the implementation of improved management strategies in recent decades [5,6]. BTB transmission is primarily caused by inhalation of infectious bacilli contained within aerosolised respiratory secretions. Following exposure, the pathogen is phagocytosed by host alveolar macrophages, which serve as key effector cells in activating the innate and adaptive immune responses required to determine the outcome of infection [7].

The immune response to $M$. bovis is similar to that elicited by $M$. tuberculosis infection in humans. Infected macrophages secrete several NF- $\mathrm{kB}$-inducible inflammatory cytokines that initiate and regulate an adaptive immune response characterised by the release of IFN- $\gamma$ from T-cells [8]. IFN- $\gamma$ activates microbicidal activity in infected macrophages and also promotes the sequestration of the pathogen in granulomas-organised complexes of immune cells consisting of lymphocytes, noninfected macrophages, dendritic cells and neutrophils that contain mycobacterial-infected macrophages and prevent the spread of bacilli to other tissues [9-11].

In many cases, however, mycobacterial pathogens can evade the host immune response and persist within alveolar macrophages resulting in lengthy subclinical phases of infection that can lead to immunopathology and disease dissemination. Pathogen survival in alveolar macrophages is achieved through a diverse range of mechanisms including the inhibition of phagosome maturation and the suppression of key immuno-regulatory pathways that mediate the host immune response to infection $[12,13]$. Consequently, analysis of the macrophage transcriptome in response to $M$. bovis infection can offer a deeper understanding of the cellular processes governing pathogen-macrophage interactions and how modulation of these cellular pathways underlie progression to active BTB. Furthermore, identification of transcriptional markers of infection may enable the development of novel diagnostics for BTB, providing new tools for disease management $[14,15]$.

The completion of an annotated Bos taurus reference genome sequence, together with developments in high-throughput transcriptomic technologies, such as immuno-specific and pan-genomic microarrays, have enabled detailed functional genomic investigation of the bovine host response to mycobacterial infections [16-21]. However, the recent advent of RNA sequencing (RNAseq) technologies offers unprecedented opportunities for gene expression analysis previously unavailable for microarray technology, including unbiased whole-transcriptome profiling, the analysis of sense and antisense transcription, the characterisation of new classes of RNA, and the identification of novel mRNA splice variants [22,23]. Furthermore, the digital nature of RNA-seq data provides a more precise and sensitive method to map and quantify RNA transcripts compared to the analog data generated by microarray technologies [24].

Previously, we used the pan-genomic high-density Affymetrix ${ }^{\circledR}$ GeneChip ${ }^{\circledR}$ Bovine Genome Array to compare temporal changes in gene expression profiles in RNA extracted from $M$. bovis-infected and non-infected control bovine monocyte-derived macrophages (MDM) purified from seven age and sex-matched HolsteinFriesian females at intervals of 2, 6 and $24 \mathrm{~h}$ postinfection [20]. We demonstrated that the number of differentially expressed genes increased sequentially at each time point post-infection, with the highest number of differentially expressed genes observed $24 \mathrm{~h}$ postinfection [20].

To gain a deeper understanding of the transcriptional changes induced $24 \mathrm{~h}$ post-infection with $M$. bovis, we have used strand-specific RNA-seq technology for the present study, to analyse the transcriptomes of the infected and non-infected control MDM samples generated by us previously [20]. A list of differentially expressed genes was generated by comparing the MDM transcriptomes from the infected and non-infected control samples, and these genes were further analysed using the Ingenuity ${ }^{\circledR}$ Systems Pathway Analysis Knowledge Base to identify macrophage cellular pathways underlying $M$. bovis infection in vitro. Finally, the list of differentially expressed genes generated from analysis of the RNA-seq data was compared to results from a comparable microarray experiment published by our group [20].

\section{Results}

\section{Summary statistics for the RNA-seq data}

All 14 RNA-seq libraries were sequenced across seven lanes of one Illumina ${ }^{\circledR}$ flow cell with a mean of 25.5 million reads (range: 20.7 million to 29.3 million reads) generated per lane. Deconvolution and filtering of sequence reads to remove adapter-dimer sequences yielded a mean of 11.3 million reads (range: 2.7 million to 18.0 million reads) per individual RNA-seq library. Subsequent alignment of the filtered RNA-seq reads to the B. taurus reference genome (Btau 4.0.63 genome release) yielded a mean of 7.2 million reads $(63.6 \%)$ for each RNA-seq library that mapped to unique locations in the bovine genome; a mean of 3.3 million reads (29.3\%) for each library that mapped to multiple locations in the genome; and a mean of 0.8 million reads for each library (7.1\%) that did not map to any genome location (Figure 1A). The number of reads per individual RNA-seq library is provided in Additional file 1: Table S1. 


\section{$\begin{array}{ll}\text { A. Reads alignment with TopHat } & \text { B. Reads assignment with HTseq }\end{array}$}

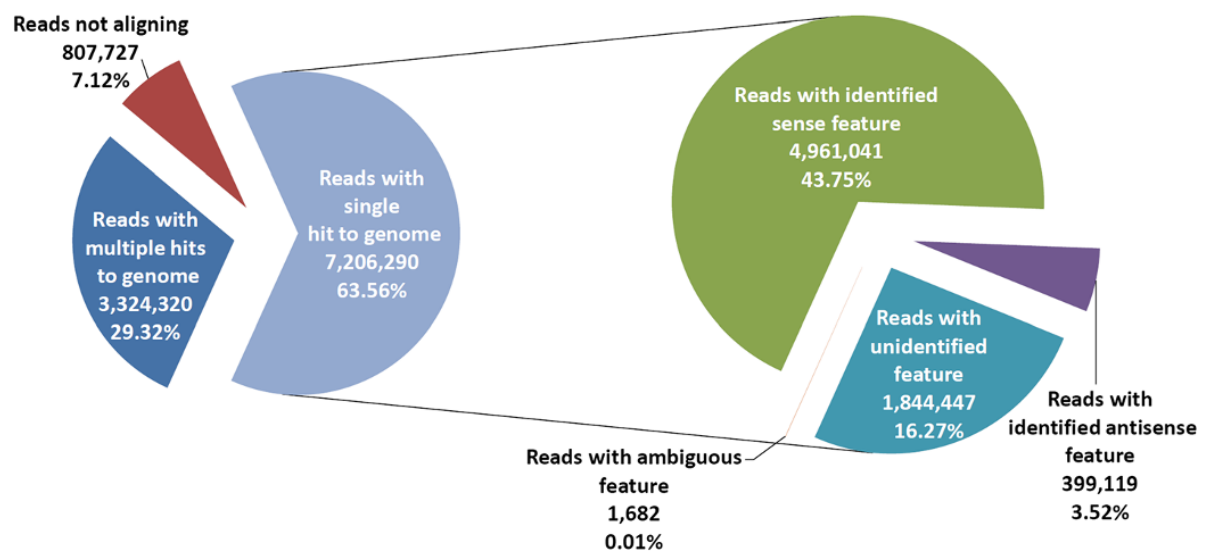

Figure 1 Apportionment of reads mapping to unique and multiple locations in the $B$. taurus reference genome. A) Pie chart showing the mean number and percentage of reads that aligned to unique location and multiple locations in the $B$. taurus reference genome using the TopHat splice junction mapper. B) Pie chart showing the mean number and percentage of uniquely mapped reads assigned to ambiguous gene features (i.e. reads that map to overlapping gene sequences), unidentified gene features (i.e. reads that map to the genome that have no gene annotation) and identified features (i.e. known gene sequences) based on sense strand and antisense strand data using the HTSeq package.

Further analysis of the individual library reads mapping to unique locations in the B. taurus reference genome (7.2 million reads) revealed that a mean of 5.3 million reads $(73.6 \%)$ aligned to exonic regions. 1.6 million $(22.2 \%)$ and 0.2 million $(0.3 \%)$ reads were associated with exon-3' UTR and exon-5' UTR sequences, respectively; 1.4 million reads (19.4\%) mapped to intergenic locations and 0.4 million reads (5.5\%) mapped to intronic regions (Figure 2). The filtered sequence reads for all deconvoluted libraries were also aligned to the complete genome sequence of the M. bovis AF2122/97 strain
(GenBank accession number NC_002945.3) to assess the presence of mycobacterial RNA contamination in individual libraries-a mean of 234 reads (0.004\%) mapped to locations in the $M$. bovis genome. The reads that mapped to the $M$. bovis genome did not map to the B. taurus reference genome. Following preliminary filtering and quality checks, only sequence reads that mapped to unique locations in the B. taurus reference genome were used for downstream bioinformatics and IPA analyses.

Quantification of the number of reads that exclusively mapped to genes with bovine Ensembl IDs was performed

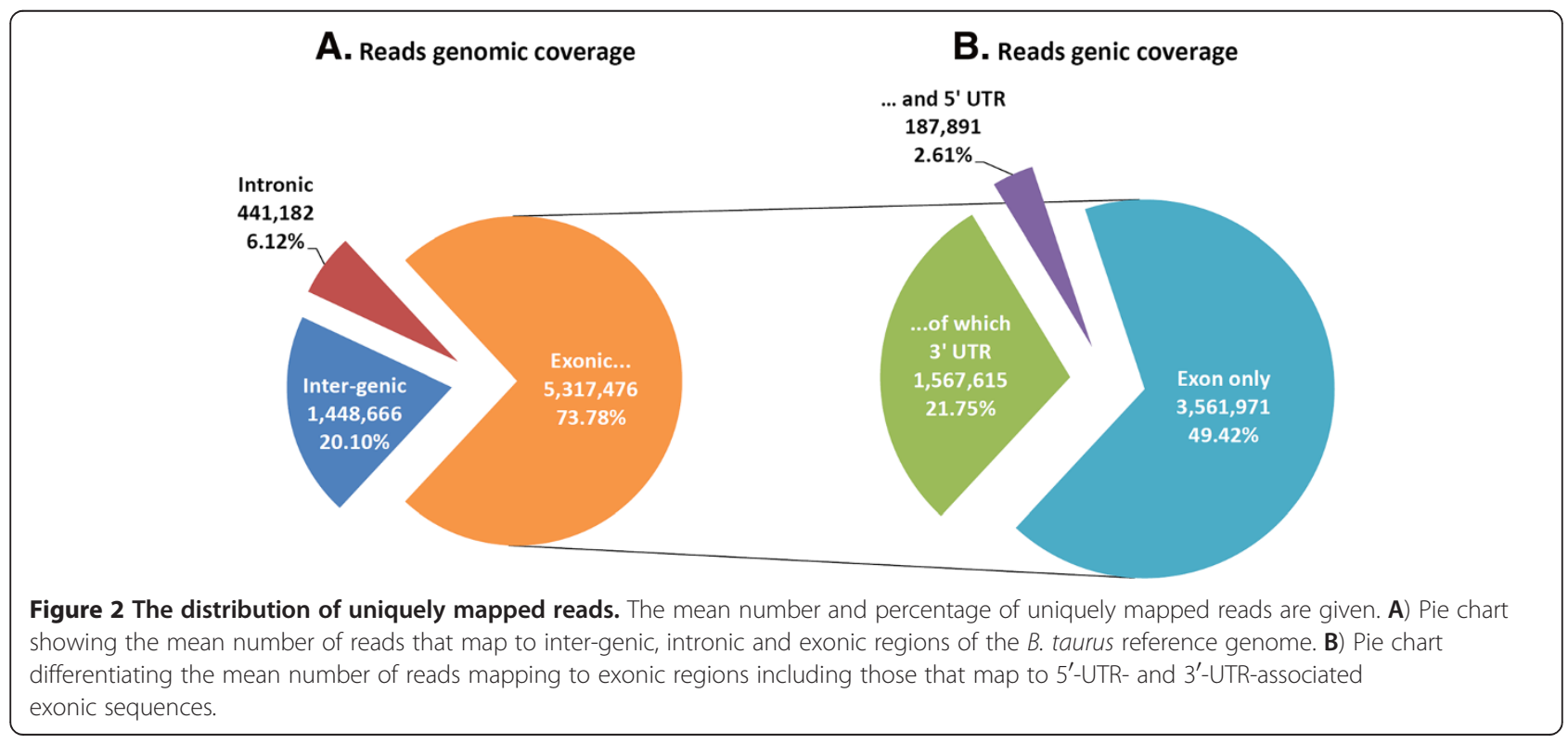


using the HTseq-count software package. This analysis demonstrated that a mean of 5.0 million reads (43.8\%) per library mapped to Ensembl gene IDs based on sense strand sequence information, with a mean of 0.4 million sequence reads for each library (3.5\%) mapping to Ensembl gene IDs based on antisense strand sequence information; only these two sets of reads were used to separately derive gene expression values for sense and antisense strand transcription, respectively. Of the remaining reads -which were not used to derive gene expression valuesa mean of 1,600 reads $(0.01 \%)$ for each library were associated with multiple Ensembl gene IDs, while a mean of 1.8 million reads for each library (16.3\%) were not associated with Ensembl gene IDs (Figure 1B).

Prior to multi-dimensional scaling (MDS) and differential gene expression analysis, density plots (Additional file 2: Figure S1) displaying the number of sequence reads per gene were constructed and analysed. Two RNA-seq libraries comprising a control and $M$. bovis-infected sample from the same animal (animal number 700; Additional file 1: Table S1) showed skewed distributions compared to all other libraries; consequently these samples were removed from all further downstream analyses. All downstream analyses, including differential gene expression, IPA analysis based on sense and antisense strand data, and technical comparisons between RNA-seq and microarray platforms were performed using data from the remaining six animals (i.e. 12 RNA-seq libraries).

\section{Gene expression and IPA analysis of sense strand transcription}

Analysis of the gene coverage using reads mapping to unique locations of the $B$. taurus reference genome based exclusively on sense strand sequence information, showed that of the 25,669 annotated B. taurus genes, 15,422 genes $(60.1 \%)$ had at least one sequence read count (i.e. one mapped read) in at least one library. The 15,422 detectable genes were further filtered by removing lowly expressed genes. Consequently, only genes displaying more than one count per million reads for at least three libraries were used for subsequent analyses. This yielded 11,131 genes (43.4\% of annotated B. taurus genes) that were suitable for downstream analyses.

Prior to differential gene expression analysis, the data from the 11,131 filtered genes were used for MDS analysis (Figure 3). MDS analysis demonstrated that MDM samples were differentiated according to treatment status (i.e. infected versus non-infected control samples) along dimension 1 , while dimension 2 separated the MDM samples according to animal ID.

Statistical analysis of all 11,131 genes that passed the filtering process identified a total of 2,584 differentially expressed genes (adjusted $P$-value $\leq 0.05$ ), of which 1,392 were upregulated and 1,192 were downregulated in the $M$. bovis-infected MDM relative to the non-infected control MDM. In addition, expression fold-change values were markedly higher for upregulated genes compared to

(based on RNA-seq data)

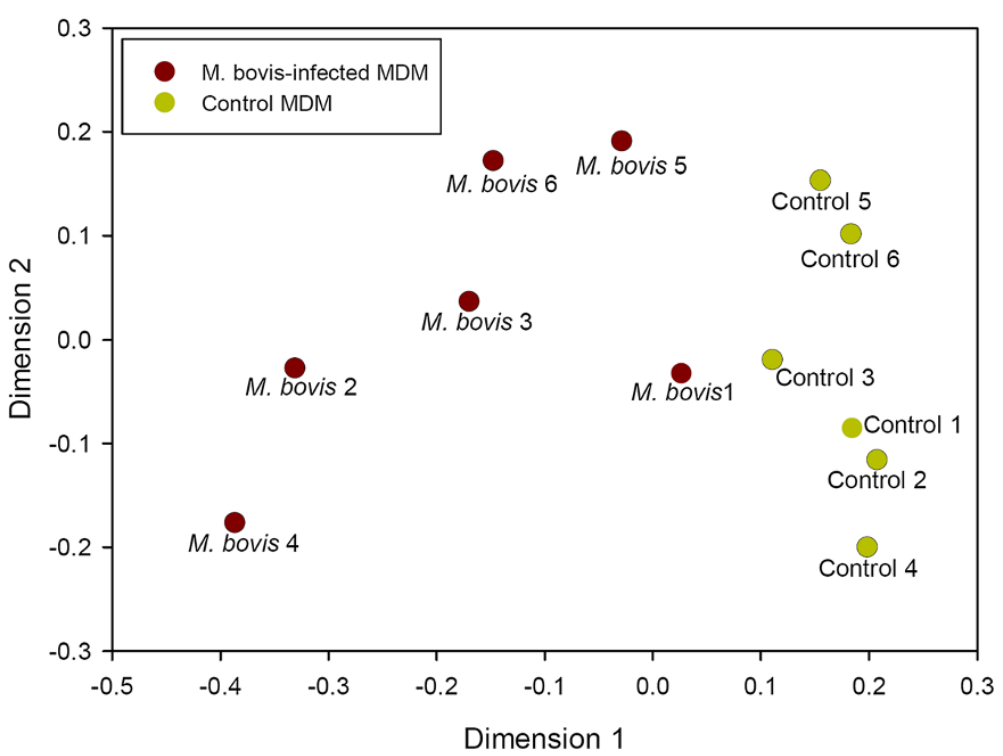

Figure 3 Multi-dimensional scale plot of all $M$. bovis-infected and control samples based on RNA-seq sense data. Dimension 1 and dimension 2 separate all 12 RNA-seq libraries based on the expression value of the 11,131 genes (based on RNA-seq sense strand data only) that passed all data filtering criteria prior to differential gene expression analysis. 
downregulated genes. A list of the differentially expressed genes based on sense strand data is presented in Additional file 3: Table S2.

Among the most upregulated genes based on expression fold-change (Table 1), were the adrenergic, beta-3-, receptor gene [ADRB3] ( $2^{\text {nd }}$ ranked upregulated gene); the interleukin $17 \mathrm{~A}$ gene $[I L 17 \mathrm{~A}]\left(3^{\text {rd }}\right.$ ranked); the serum amyloid A 3 gene [SAA3] ( $4^{\text {th }}$ ranked); and the mammary serum amyloid A3.2 gene [M-SAA3.2] (5 ${ }^{\text {th }}$ ranked). The first ranked upregulated gene is not described here as it encodes an uncharacterized protein. The most downregulated genes were the gamma-aminobutyric acid (GABA) B receptor, 2 gene [GABBR2] ( $1^{\text {st }}$ ranked downregulated gene); the cadherin 26 gene [ $C D H 26]$ ( ${ }^{\text {nd }}$ ranked); the solute carrier family 16, member 12 (monocarboxylic acid transporter 12) gene [SLC16A12] ( $3^{\text {rd }}$ ranked); the Fas apoptotic inhibitory molecule 2 gene [FAIM2] ( $4^{\text {th }}$ ranked); and the SH3 domain and tetratricopeptide repeats 2 gene [SH3TC2] $\left(5^{\text {th }}\right.$ ranked).

Functional categorisation of the 2,584 differentially expressed genes using IPA revealed an enrichment of genes with roles in immunology, cell development and proliferation, and apoptosis (Additional file 4: Table S3). IPA was also used to identify canonical pathways enriched for differentially expressed genes, many of which were had immune and apoptotic functions. (Additional file 5: Table S4). Based on the well-documented role of apoptosis in the host response to mycobacterial infection [25] the Death receptor signalling canonical pathway was overlaid with RNA-seq gene expression results and is presented in Figure 4.

Gene expression analysis of antisense strand read data Analysis of the RNA-seq reads that mapped to antisense strand gene sequences revealed that 13,796 genes of the 25,669 annotated B. taurus genes had at least one sequence read count (i.e. one mapped read count) for at least one library. Filtering out of lowly expressed genes (as detailed for the sense strand data above) led to the exclusion of 6,954 genes, leaving a total of 6,842 genes for differential expression analysis based of antisense strand data. 757 of the genes were differentially expressed (adjusted $P$-value $\leq 0.05$ ), of which 558 showed increased expression and 199 showed decreased expression in the $M$. bovis-infected MDM relative to the non-infected control MDM. As with the sense strand data, the fold-change in expression for the genes showing increased expression was markedly higher than that for the genes decreasing in expression. A list of significant differentially expressed genes derived from the antisense strand expression data is presented in Additional file 6: Table S5. Additional file 7: Figure S2 shows the distribution of sense and antisense reads that mapped to the spectrin, beta, erythrocytic gene $(S P T B)$. As these reads do not map to the exact same locations in the $S P T B$ gene, we are confident that they represent actual antisense transcripts based on the current annotation of the $B$. taurus reference genome and are not due to technical artefacts introduced during RNA-seq library preparation.

A systematic comparison was performed between significantly differentially expressed genes detected using the sense strand RNA-seq data $(n=2,584)$ and those identified from the antisense strand data $(n=757)$. This generated a list of 694 differentially expressed genes that were common to both the sense strand and the antisense strand data sets. The common 694 genes were further subdivided according to the direction of expression (i.e. up- or downregulated) in the $M$. bovis-infected MDM relative to the control MDM. 520 genes displayed an increase in expression in both the sense and antisense strand data sets, while 173 genes showed decreased expression from both strands. In

Table 1 List of the top five ranking up- and downregulated genes based on the RNA-seq sense strand data

\begin{tabular}{|c|c|c|c|c|c|c|}
\hline Rank & $\begin{array}{l}\text { Direction of } \\
\text { expression }\end{array}$ & Ensembl Gene ID & $\begin{array}{l}\text { Gene } \\
\text { symbol }\end{array}$ & Gene name & $\begin{array}{l}\log _{2} \text { fold } \\
\text { change }\end{array}$ & Adjusted $P$-value \\
\hline 1 & Upregulated & ENSBTAG00000023064 & Not available & Uncharacterized protein & 8.41 & $7.62 \times 10^{-21}$ \\
\hline 2 & Upregulated & ENSBTAG00000017981 & ADRB3 & adrenergic, beta-3-, receptor & 8.01 & $2.48 \times 10^{-17}$ \\
\hline 3 & Upregulated & ENSBTAG00000002150 & IL17A & interleukin 17A & 7.71 & $1.37 \times 10^{-14}$ \\
\hline 4 & Upregulated & ENSBTAG00000022396 & SAA3 & serum amyloid $\mathrm{A} 3$ & 7.50 & $7.67 \times 10^{-114}$ \\
\hline 5 & Upregulated & ENSBTAG00000010433 & M-SAA3.2 & mammary serum amyloid A3.2 & 6.95 & $5.26 \times 10^{-41}$ \\
\hline 1 & Downregulated & ENSBTAG00000013810 & GABBR2 & $\begin{array}{l}\text { gamma-aminobutyric acid (GABA) } \\
\text { B receptor, } 2\end{array}$ & -6.67 & $5.49 \times 10^{-08}$ \\
\hline 2 & Downregulated & ENSBTAG00000020261 & $\mathrm{CDH} 26$ & cadherin 26 & -5.68 & $4.25 \times 10^{-18}$ \\
\hline 3 & Downregulated & ENSBTAG00000004662 & SLC16A12 & $\begin{array}{l}\text { solute carrier family } 16, \text { member } 12 \\
\text { (monocarboxylic acid transporter } 12 \text { ) }\end{array}$ & -5.52 & $3.01 \times 10^{-16}$ \\
\hline 4 & Downregulated & ENSBTAG00000017504 & FAIM2 & Fas apoptotic inhibitory molecule 2 & -4.40 & $6.53 \times 10^{-12}$ \\
\hline 5 & Downregulated & ENSBTAG00000017151 & SH3TC2 & SH3 domain and tetratricopeptide repeats 2 & -4.28 & $4.29 \times 10^{-10}$ \\
\hline
\end{tabular}

List of the top most upregulated and downregulated genes in $M$. bovis-infected samples relative to the control samples are shown (ranked by fold change) based on RNA-seq sense strand data. 


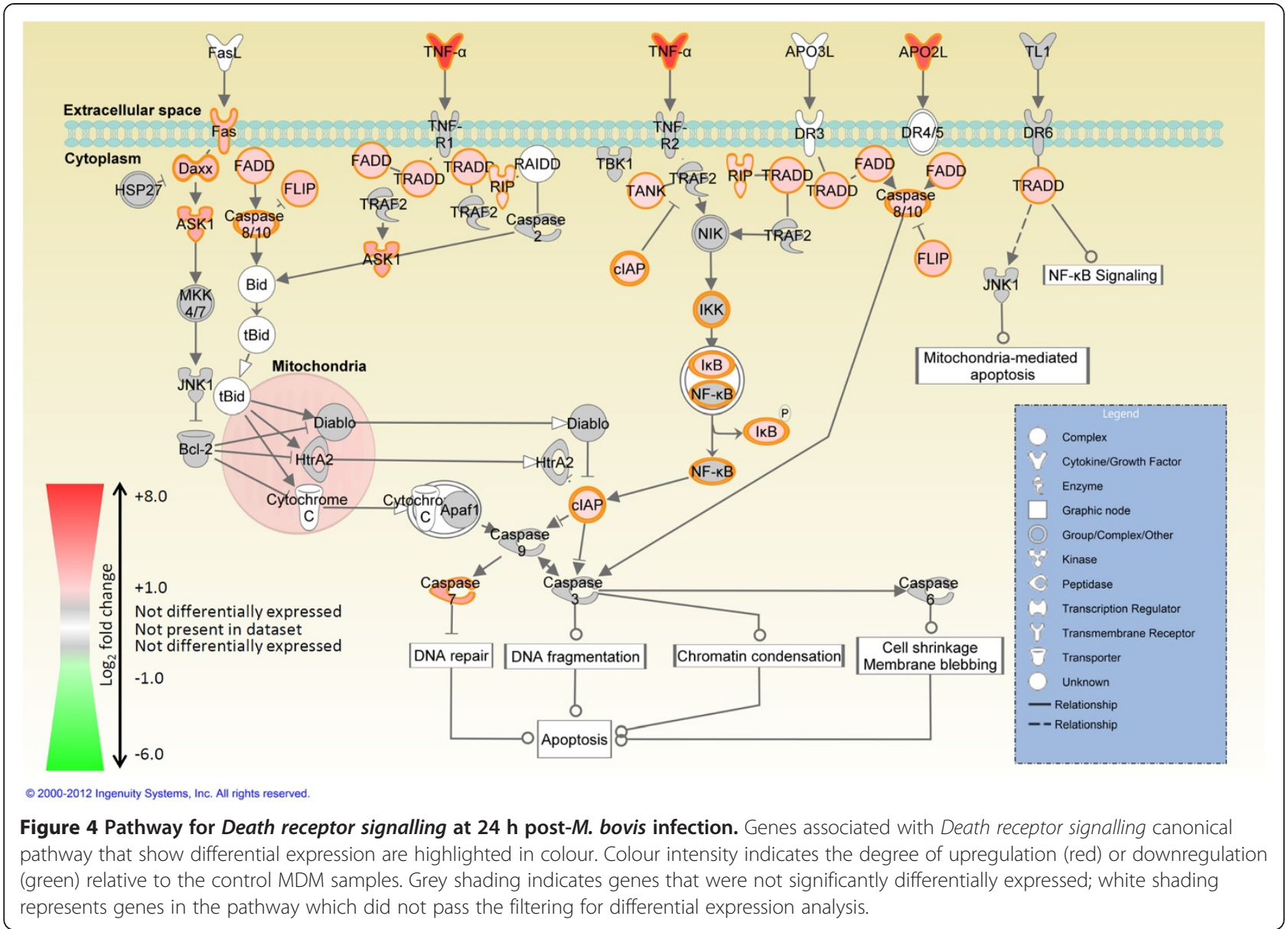

addition, no gene displayed increased expression from the sense strand and decrease expression from the antisense strand. Finally, one gene showed decreased expression from the sense strand and increased expression from the antisense strand (Figure 5).

Functional categorisation of the 757 differentially expressed genes based on antisense strand data using IPA revealed an enrichment of genes with roles in immunology, cell development and proliferation, and response to infection (Additional file 8: Table S6). Four of the top five ranking GO categories identified from the antisense and sense strand data were identical (Additional file 4: Table S3 and Additional file 8: Table S6). Similarly, IPA analysis of the antisense strand data generated a list of canonical pathways with immune related function (Additional file 9: Table S7). Four of the five top-ranking canonical pathways identified from the antisense and sense strand data were identical (Additional file 5: Table S4 and Additional file 9: Table S7).

\section{Comparison of differential gene expression profiles obtained from RNA-seq and microarray platforms} The total RNA samples extracted from $M$. bovis-infected and non-infected control MDM samples described here,

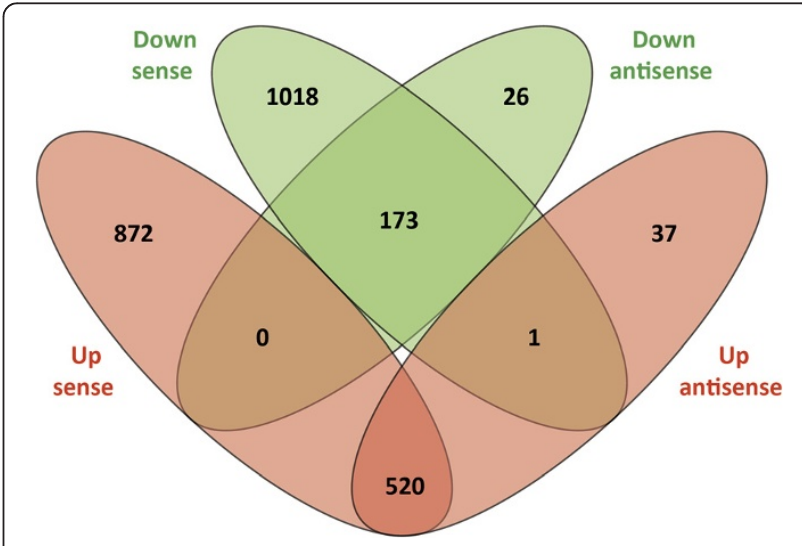

Figure 5 Venn diagrams showing comparison of RNA-seq sense versus antisense strand differential gene expression. Venn diagram showing the comparison of the direction of differential expression based on sense and antisense strand data. Upregulated and downregulated genes are shaded red and green, respectively. 
were previously used by us for gene expression profiling with the Affymetrix ${ }^{\circledR}$ GeneChip ${ }^{\circledR}$ Bovine Genome Array [20]. This array contains 24,027 probe sets representing more than 23,000 transcripts and includes approximately 19,000 UniGene clusters [26].

In order to directly compare gene expression profiles from these previously generated microarray data with the RNA-seq data generated in the current study, a number of steps were performed. Firstly, we removed the same two samples which were excluded from the RNA-seq data analysis (i.e. the non-infected control and $M$. bovis-infected MDM-extracted RNA from animal number 700), then re-analysed all microarray data using all 12 RNA samples from the remaining six animals. Secondly, we mapped Affymetrix ${ }^{\circledR}$ GeneChip ${ }^{\circledR}$ Bovine Genome Array probes to genes with bovine Ensembl gene IDs based on data from the $B$. taurus reference genome. Of the 24,072 probe sets represented on the array, 11,790 probe sets passed filtering; with 8,560 unique probe sets mapping to a $B$. taurus Ensembl gene ID, representing 6,807 unique genes. Prior to differential gene expression analysis, the data from the 11,790 filtered probes was used for MDS analysis (Additional file 10: Figure S3). As with the data from the RNA-seq , the MDS analysis demonstrated that MDM samples were differentiated according to treatment status (i.e. infected versus non-infected control samples) along dimension 1, while dimension 2 separated the MDM samples according to animal ID. It is important to note that joint inspection of Figure 3 and Additional file 10: Figure S3 shows that the samples are spatially arranged in a very similar pattern for the two different gene expression platforms.

\section{Comparison of the RNA-seq and microarray dynamic ranges}

To assess the dynamic range of the RNA-seq and microarray data sets, we analysed the $\log _{2}$ microarray intensities and the $\log _{2}$ reads per kilobase per million mapped reads (RPKM) values for all genes that passed the filtering criteria. From this, we calculated the dynamic range of the microarray by subtracting the gene with the highest mean $\log _{2}$ intensity (ENSBTAG00000018784 $[C T S Z] ; \log _{2}$ intensity $=15.48$ ) from the gene with the lowest mean $\log _{2}$ intensity (ENSBTAG00000002021 [BNIP1]; $\log _{2}$ intensity $=2.03$ ), yielding a $\log _{2}$ dynamic range of 13.45. We then calculated the dynamic range of RNA-seq by subtracting the gene with the highest mean $\log _{2}$ RPKM (ENSBTAG00000043561 [COX1]; $\log _{2}$ RPKM $=12.60$ ) from the gene with the lowest mean $\log _{2}$ RPKM (ENSBTAG00000001325 [UPB1]; $\log _{2}$ RPKM = -6.83), yielding a $\log _{2}$ dynamic range of 19.43 . Our analysis therefore shows that the dynamic range for RNA-seq is greater than the microarray. This method for dynamic range calculations of gene expression data has been previously described by Chen and colleagues [27]. Furthermore, it is important to note that raw RPKM values are proportions; consequently, values less than 1.0 yield negative values when $\log _{2}$-transformed.

To ensure that differences in the dynamic ranges were not due to contrasting normalisation procedures, we determined the dynamic range of the RNA-seq data following a quantile normalisation strategy, which is comparable to the quantile normalisation used for the microarray. This analysis yielded a $\log _{2}$ dynamic range for the RNA-seq data of 20.01, which further supports a greater dynamic range for the RNA-seq platform compared to the microarray.

\section{Comparison of high and low transcript expression from RNA-seq and microarray data}

For this analysis, we compared $\log _{2}$ reads per kilobase (of transcript sequence) per million reads (RPKM) with the $\log _{2}$ raw microarray hybridisation intensity probe signals. Genes with alternative transcripts-for which, a definitive gene length necessary for RPKM calculations could not be derived-were omitted from this analysis. Spearman rank correlation analysis was first performed for a total of 5,560 common transcripts that passed the RNA-seq and microarray filtering criteria (detailed in Methods). This analysis was performed separately for the non-infected control and infected MDM samples. We observed a significant $(P \leq 0.01)$ Spearman correlation coefficient of 0.66 and 0.64 for the infected and the control samples, respectively. These results suggest that there is a positive relationship between the microarray and RNAseq data sets such that: (1) transcripts that yield a high intensity value on the microarray also yield a high read count based on the RNA-seq platform; and (2) transcripts that yield a low intensity value on the microarray also yield a low read count based on the RNA-seq platform.

We next binned the transcripts into groups of highly, moderately and lowly expressed transcripts (based on the microarray data), with an approximately equal number of transcripts in each bin. We observed the greatest correlation between the highly expressed transcripts $(r=0.551$ for infected MDM; $r=0.535$ for control MDM; $P \leq 0.01)$, followed by the lowly expressed transcripts $(r=0.302$ for infected MDM; $r=0.327$ for control MDM; $P \leq 0.01$ ) followed by the moderately expressed transcripts $(r=0.261$ for infected MDM; $r=0.217$ for control MDM; $P \leq 0.01$ ). These results suggest that the correlation between the two platforms is better for highly abundant transcripts compared to moderately and lowly abundant transcripts. 


\section{Comparison of expression fold-change and difference in expression value between treatments for RNA-seq and microarray data}

To investigate if the greatest fold-change in expression was largely observed in genes with low levels of expression, we compared: (1) the $\log _{2}$ fold-change with the $\log _{2}$ differences in counts per million reads (CPM) between the control and infected MDM groups for the RNA-seq platform; and (2) the $\log _{2}$ fold-change with the $\log _{2}$ differences in hybridisation intensity between the control and infected MDM groups for the microarray platform. We hypothesised that if transcripts with the lowest expression gave the highest fold-change values, then a negative correlation between $\log _{2}$ fold-change and $\log _{2}$ differences for genes displaying increased expression post-infection. Reciprocally, a positive correlation would be expected between $\log _{2}$ fold-change and $\log _{2}$ differences for genes displaying decreased expression post-infection.

For RNA-seq, this analysis was based on 11,131 genes that passed the filtering criteria; of which, 5,377 displayed increased expression and 5,754 genes displayed decreased expression following MDM infection. We observed Spearman correlation coefficients of 0.536 and -0.394 for the genes displaying increased and decreased expression following infection, respectively $(P \leq 0.001)$. These correlation coefficients contrast with those expected based on our hypothesis described above; therefore, we conclude that there is no obvious relationship between gene expression level and fold-change.

For the microarray, our analysis was based on 11,665 genes that passed the filtering criteria; of which, 5,020 displayed increased expression and 6,645 genes displayed decreased expression following MDM infection. Spearman correlation coefficients of 0.378 and -0.128 were observed for the genes displaying increased and decreased expression following infection, respectively $(P \leq 0.001)$. Again, we conclude that there is no obvious relationship between gene expression level and fold-change.

\section{Comparison of fold-change in expression from RNA-seq and microarray data}

We compared $\log _{2}$ fold-changes in expression for a total of 6,183 genes common to both RNA-seq and the microarray that passed the data filtering criteria. This analysis encompassed, but was not restricted, to the differentially expressed genes identified from both data sets, the description and analysis of which is presented below. Note the number of genes analysed here was greater than the total number of transcripts analysed for transcript expression comparison as RPKM could not be calculated for genes with alternative transcripts as stated above (so reducing the number of comparable genes in the analysis above). The Spearman correlation coefficient of $\log _{2}$ fold-changes between the RNA-seq and microarray was $0.858(P \leq 0.01)$, indicating that the fold-change magnitudes for the platforms were very similar.

\section{Comparison of the number and fold-change of differentially expressed genes identified from RNA-seq and microarray}

Subsequent analysis of the microarray data showed that 2,015 unique genes were differentially expressed, with 917 genes and 1,098 genes displaying up- and downregulation, respectively, in the infected MDM relative to the control MDM (FDR $\leq 0.05$; Additional file 11: Table S8). Comparison of the differentially expressed genes with Ensembl gene IDs identified from the microarray and RNA-seq sense strand data sets revealed 964 differentially expressed genes displaying the same direction of expression that were common to both the microarray and RNA-seq data sets; these comprised 607 upregulated and 357 downregulated common genes. One gene was found respectively downregulated and upregulated in the microarray and RNA-seq sense strand data sets (Additional file 12: Table S9). Of the remaining differentially expressed genes detected, 1,050 genes (comprising 310 upregulated genes and 740 downregulated genes) were unique to the microarray data set, while 1,619 genes (comprising 784 upregulated genes and 835 downregulated genes) were unique to the RNA-seq data set (Figure 6). The differentially expressed genes that were common to both platforms and displayed the same direction of expression, represent $47.8 \%$ (i.e. 964/2,015 genes) and 37.3\% (i.e. 964/2,584 genes) of all differentially expressed genes detected by microarray and RNA-seq analysis, respectively.

Spearman rank analysis of the $\log _{2}$ RPKM values (RNA-seq data) with the $\log _{2}$ hybridisation intensity

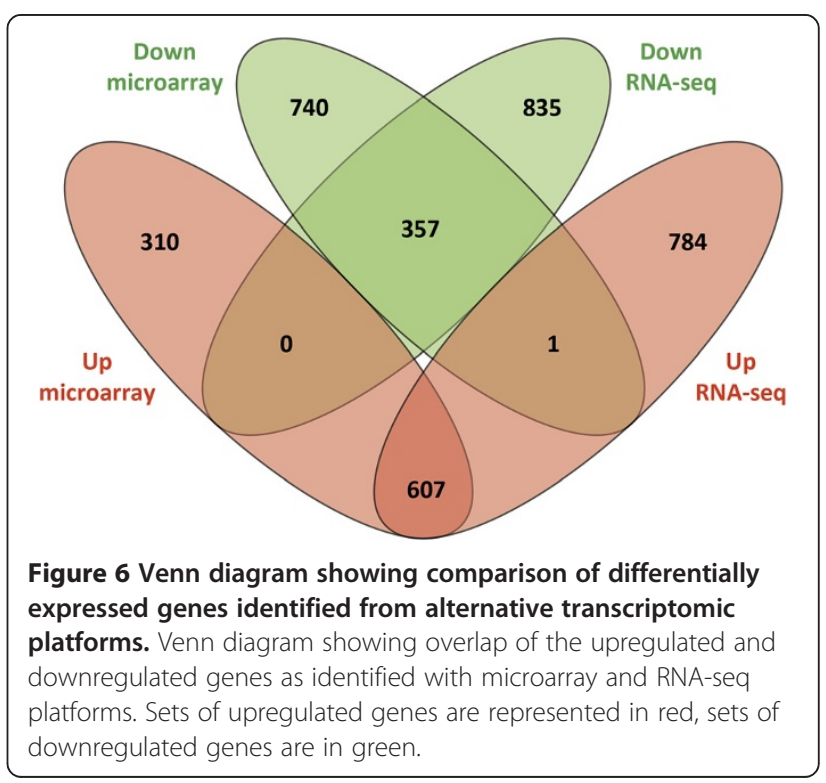


signals (microarray data) for all 964 differentially expressed genes that were common to both platforms and had the same direction of expression revealed a correlation coefficient of $r=0.713$ for infected MDM and $r$ $=0.660$ for control MDM $(P \leq 0.01)$, indicating that there is a positive relationship between raw signal intensities for each platform/treatment group. Finally, comparison of the $\log _{2}$ fold-change in gene expression between the two platforms for these 964 genes yielded a correlation coefficient of $r=0.929$ ( $P \leq 0.001)$.

To investigate the effect of platform dynamic range on the number differentially expressed genes unique to each platform we plotted and compared the density of the $\log _{2}$ of the mean hybridisation intensity and the density of the $\log _{2}$ of the mean CPM for the differentially expressed genes unique to each platform and for the differentially expressed genes common to both platforms. This analysis showed that differentially expressed genes uniquely detected by RNA-seq are largely characterised by low levels of expression for both the control and infected MDM groups. This pattern was not observed for the microarray data (Additional file 13: Figure S4). This analysis shows that the sensitivity of RNA-seq for the detection of lowly expressed transcripts is greater than the microarray.

\section{IPA analysis of differentially expressed genes common to both microarray and RNA-seq}

To validate the IPA analysis performed using differentially expressed genes based on RNA-seq sense strand data only, we uploaded the 965 differentially expressed genes common to both RNA-seq and the microarray into IPA (964 genes plus the one gene that displayed reciprocal expression for the two platforms). We hypothesised that the 965 common genes were more likely to reflect true biological changes induced following infection with $M$. bovis, compared to the differentially expressed genes identified by either platform independently. This approach has been previously used to validate GO category analysis in a study where RNA-seq and microarray data were available for the same samples [28]. Of the 63 GO categories identified for the common differentially expressed genes, 56 (88.9\%) and 60 (95.2\%) were observed in the RNA-seq and microarray IPA data sets, respectively (Additional file 4: Table S3, Additional file 14: Table S10 and Additional file 15: Table S11). Similarly, of the 198 canonical pathways identified for the common differentially expressed genes, $160(80.8 \%)$ and $166(83.8 \%)$ were observed in the RNA-seq and microarray IPA data sets, respectively (Additional file 5: Table S4, Additional file 16: Table S12 and Additional file 17: Table S13). This analysis suggests that meaningful biological interpretation of data from either RNA-seq or microarrays is possible.

\section{Validation of differentially expressed genes using real time quantitative reverse transcription PCR}

For method validation purposes, we quantified a panel of 16 genes via real time quantitative reverse transcription PCR (qRT-PCR) using the same MDM-extracted RNA samples that were used for the RNA-seq and microarray platforms as described above. The methods used for real time qRT-PCR analysis of these genes have been described by us elsewhere [20]. Using these data, we estimate the dynamic range of the real time qRTPCR platform has a $\log _{2}$ dynamic range of 15 -this is based on $C_{t}$ value ranges between 20 and 35 for a number of cytokine genes.

Real time qRT-PCR analysis showed that 12 of these genes (CCL4, CCL5, CCL20, CD40, CFB, CXCL2, IL15, IL1B, IL6, IRF1, NFKB2 and TNF) were upregulated, while two genes (AREGB and FOS) were downregulated in the infected MDM $(P \leq 0.05)$. The remaining two genes, PIK3IP1 and SPRY2 were not differentially expressed based on analysis of the real time qRT-PCR data.

We compared the real time qRT-PCR expression profiles of these 16 genes with their expression profiles from RNA-seq and the microarray data based on the analyses performed in the current study. Ten of the 12 upregulated genes (as determined by real time qRT-PCR analysis) were also upregulated in the RNA-seq and microarray platforms-only the IL15 and IRF1 genes were not differentially expressed based on microarray and RNA-seq results, respectively. Two genes (AREGB and FOS) were shown to be downregulated across all three gene expression technologies, while the remaining two genes (PIK3IP1 and $S P R Y 2)$ were shown to be downregulated based on results generated from microarray and RNA-seq only. Pairwise comparisons of the analytical platforms used across all 16 genes yielded concordances of $87.50 \%$ for the microarray and RNA-seq comparison, $81.25 \%$ for the real time qRTPCR and RNA-seq comparison and $81.25 \%$ for the microarray and the real time qRT-PCR. This is summarised in Table 2 .

\section{Discussion}

Analysis of the host transcriptome in response to mycobacterial infection has greatly enhanced our knowledge of the immunological mechanisms and cellular pathways that underlie initial infection, disease progression and ultimately active disease. These investigations have been underpinned by continual improvements in the technologies used for functional genomics and the bioinformatics methods for data analysis-particularly for microarray technologies, which have enabled analyses of immunespecific and pan-genomic gene expression patterns following infection [15,17-21,29]. Despite notable progress in understanding the molecular basis of host-mycobacteria interactions during infection, microarrays are not without 
Table 2 Comparison of fold-changes in gene expression based on RNA-seq, microarray and real time qRT-PCR results

\begin{tabular}{|c|c|c|c|c|c|}
\hline Gene symbol & Gene name & Gene description & $\begin{array}{l}\text { RNA-seq fold- } \\
\text { change }\end{array}$ & $\begin{array}{l}\text { Array fold- } \\
\text { change }\end{array}$ & $\begin{array}{l}\text { Real time qRT-PCR } \\
\text { fold-change }\end{array}$ \\
\hline CCL4 & $\begin{array}{l}\text { Chemokine (C-C motif) } \\
\text { ligand } 4\end{array}$ & A proinflammatory and chemotactic chemokine & 40.02 & 10.53 & 26.51 \\
\hline CCL5 & $\begin{array}{l}\text { Chemokine (C-C motif) } \\
\text { ligand } 5\end{array}$ & $\begin{array}{l}\text { A proinflammatory chemokine involved in the } \\
\text { chemotaxis of monocytes and T-helper cells }\end{array}$ & 17.43 & 14.68 & 19.12 \\
\hline CCL2O & $\begin{array}{l}\text { Chemokine (C-C motif) } \\
\text { ligand } 20\end{array}$ & $\begin{array}{l}\text { A chemokine involved in the chemoattraction } \\
\text { of lymphocytes and neutrophils }\end{array}$ & 67.34 & 82.33 & 45.94 \\
\hline CD40 & $\begin{array}{l}\text { CD40 molecule, TNF } \\
\text { receptor superfamily } \\
\text { member } 5\end{array}$ & $\begin{array}{l}\text { A member of the TNF-receptor superfamily. } \\
\text { Mediates the immune and inflammatory } \\
\text { responses }\end{array}$ & 13.43 & 9.99 & 12.32 \\
\hline$C F B$ & Complement factor B & $\begin{array}{l}\text { A component of the alternative pathway of } \\
\text { complement activation }\end{array}$ & 3.87 & 40.63 & 40.36 \\
\hline CXCL2 & $\begin{array}{l}\text { Chemokine (C-X-C motif) } \\
\text { ligand } 2\end{array}$ & $\begin{array}{l}\text { An immunoregulatory chemokine produced by } \\
\text { activated monocytes and neutrophils at sites of } \\
\text { inflammation }\end{array}$ & 15.72 & 4.44 & 14.12 \\
\hline IL15 & Interleukin 15 & $\begin{array}{l}\text { A cytokine that regulates } T \text { and natural killer cell } \\
\text { activation and proliferation }\end{array}$ & 3.07 & Not DE & 2.23 \\
\hline ILIB & Interleukin 1, beta & $\begin{array}{l}\text { A cytokine that mediates the inflammatory } \\
\text { response including cell proliferation, } \\
\text { differentiation and apoptosis }\end{array}$ & 34.84 & 92.28 & 41.21 \\
\hline IL6 & Interleukin 6 & $\begin{array}{l}\text { A cytokine that functions in inflammation and } \\
\text { the maturation of B cells }\end{array}$ & 39.62 & 88.47 & 55.08 \\
\hline IRFI & $\begin{array}{l}\text { Interferon regulatory } \\
\text { factor } 1\end{array}$ & $\begin{array}{l}\text { A member of the interferon regulatory } \\
\text { transcription factor family. An activator of } \\
\text { interferon alpha and beta transcription }\end{array}$ & Not DE & 10.01 & 9.74 \\
\hline NFKB2 & $\begin{array}{l}\text { Nuclear factor of kappa } \\
\text { light polypeptide gene } \\
\text { enhancer in B-cells } 2 \\
\text { (p49/p100) }\end{array}$ & $\begin{array}{l}\text { A pleiotropic transcription factor involved in } \\
\text { inflammation, immunity, differentiation, cell } \\
\text { growth and apoptosis }\end{array}$ & 5.35 & 8.68 & 5.76 \\
\hline TNF & $\begin{array}{l}\text { Tumor necrosis factor } \\
\text { (TNF superfamily, } \\
\text { member 2) }\end{array}$ & $\begin{array}{l}\text { A proinflammatory cytokine (secreted by } \\
\text { macrophages) involved in the regulation cell } \\
\text { proliferation, differentiation and apoptosis, and } \\
\text { coagulation. }\end{array}$ & 15.16 & 80.07 & 15.00 \\
\hline$A R E G B$ & Amphiregulin B & A growth-modulating glycoprotein & -3.84 & -9.70 & -5.09 \\
\hline FOS & $\begin{array}{l}\text { FBJ murine osteosarcoma } \\
\text { viral oncogene homolog }\end{array}$ & $\begin{array}{l}\text { A leucine zipper protein member of the AP-1 } \\
\text { transcription factor complex. }\end{array}$ & -3.69 & -5.50 & -3.61 \\
\hline PIK3IP1 & $\begin{array}{l}\text { Phosphoinositide-3-kinase } \\
\text { interacting protein } 1\end{array}$ & $\begin{array}{l}\text { Suppresses the activity of phosphatidylinositol-3 } \\
\text {-kinase (PI3K), a regulator of cell division }\end{array}$ & -2.06 & -1.68 & Not DE \\
\hline SPRY2 & $\begin{array}{l}\text { Sprouty homolog } 2 \\
\text { (Drosophila) }\end{array}$ & $\begin{array}{l}\text { An inhibitor of receptor tyrosine kinase } \\
\text { signalling proteins }\end{array}$ & -2.12 & -4.64 & Not DE \\
\hline
\end{tabular}

Geometric mean fold-changes of gene expression ( $M$. bovis-infected samples relative to control samples) are given for the RNA-seq sense strand, microarray and real time qRT-PCR data. Unless specified, results for each gene using the three techniques displayed significant differential expression $(P$-value $\leq 0.05)$. 'Not $D E$ ' indicates that a gene was not differentially expressed.

their limitations, including: (1) a requirement for prior DNA sequence knowledge of annotated genes for probe design; (2) indirect quantification of gene expression by hybridisation signal intensities; (3) constrained dynamic ranges that impair quantification of lowly and highly expressed gene transcripts; and (4) a limited ability to detect splice variants and novel classes of non-coding regulatory RNA [30-32].

The RNA-seq approach, which is based on ultra-high throughput sequencing of total RNA and systematic counts of all expressed transcripts, has the potential to overcome many of the limitations associated with microarray technology. In particular, RNA-seq: (1) requires no prior sequence information (however, a reference genome is normally used, but not required for mapping of raw sequence reads) [33]; (2) has a larger dynamic range and is more sensitive than microarray data because the quantification of each gene transcript is based directly on the number of reads mapping to a particular gene; and (3) may provide additional information regarding the complexity of the transcriptome, including strandspecific gene expression, identification of novel genes, 
and identification of splicing events and a wide range of non-coding RNA species $[23,34]$. Therefore, RNA-seq can provide a sensitive, unbiased and fully quantitative and qualitative transcriptomic profile of host cells following mycobacterial infection. Consequently, we have used strand-specific RNA-seq to examine the transcriptome of bovine MDM following a $24 \mathrm{~h}$ in vitro infection with $M$. bovis (multiplicity of infection [MOI] 2:1) to gain novel insights into the transcriptional changes and cellular pathways induced during the early stages of infection. In addition, as the MDM samples described here have been previously studied using the Affymetrix ${ }^{\circledR}$ GeneChip $^{\circledR}$ Bovine Genome Array, we have performed a comparison of the results generated from both analytical platforms.

\section{Summary of RNA-seq results}

For each individual RNA-seq library analysed here, we obtained a mean of 7.2 million $69 \mathrm{bp}$ reads that mapped to single unique locations in the $B$. taurus reference genome. This yielded a mean of $496.8 \mathrm{Mb}$ of sequence per individual library. Examination of the gene coverage from these uniquely mapping reads demonstrated that 15,422 genes (based only on sense strand data) from a total of 25,669 annotated bovine genes (60.1\%) gave at least one sequence read count in total. However, studies have shown that gene expression analysis using RNAseq is dependent on sequencing depth and that genes with low sequencing coverage are more susceptible to the generation of false-positive differentially expressed genes. In this regard, we only considered genes displaying more than one count per million reads in at least three libraries for differential gene expression analysis; consequently a total of 11,131 genes (43.4\% of the total bovine gene content) based on sense strand data were used in the analysis presented here. Although this stringent threshold lowers the number of genes for which differential expression can be performed, we believe that this criterion is sufficient for quantification and analysis of highly expressed genes with a corresponding reduction in the number of Type I errors due to lowly expressed genes [35-37].

\section{Sense strand gene expression and IPA analyses}

Gene expression analysis of the sense strand information in the current study detected a total of 2,584 differentially expressed genes (adjusted $P$-value $\leq 0.05$ ), of which 1,392 and 1,192 were upregulated and downregulated, respectively, in the $M$. bovis-infected MDM relative to the non-infected control MDM. This finding contrasts with previous work performed by us and others showing that mycobacterial infection in vitro and in vivo results in a higher number of downregulated genes relative to upregulated genes based on microarray analysis $[17,20,21,29,38]$. This discrepancy is most obviously explained by differences in sensitivity and technical biases between the two transcriptomic platforms used. This assertion is supported in the current study by the increased dynamic range of RNA-seq compared to the microarray platform. In agreement with previous studies, however, the expression fold-change values were markedly higher for upregulated genes compared to downregulated genes [17,19-21].

Analyses performed at the gene level revealed that all the top upregulated and downregulated genes have roles and functions involved in or related to immune response and infectious disease. One of the most highly upregulated genes identified was $A D R B 3$; the protein encoded by this gene has been shown to have functions in carbohydrate metabolism, energy reserve metabolism, positive regulation of the MAPK cascade and regulation of apoptosis [39,40]. IL17A was also upregulated and has known role in the pro-inflammatory response, expansion and recruitment of innate immune cells, production of defensins and antimicrobial peptides, and linking innate and adaptive immune responses [41]. Also upregulated was $S A A 3$, which was the most statistically significant gene in the data set, and has a major multifunctional role in the acute-phase response [42].

Among the most highly downregulated genes was $G A B B R 2$, which has multiple roles and functions, including involvement in pulmonary disorder and regulation of the coughing process [43-45]. In addition, CDH26 was downregulated and cadherins have been shown to have a role in $\mathrm{Ca}^{2+}$-dependent cell-to-cell adhesion as well as cell polarisation and cell migration [46]; however, the specific role of $C D H 26$ has not been characterised. The SLC16A12 gene was also detected, a member of a gene family containing genes recently shown to be associated with tuberculosis susceptibility in cattle [47]. FAIM2 was highly downregulated, a gene with a role in the inhibition of apoptosis, a key process associated with mycobacterial infection of macrophages [25,48]. Finally, SH3TC2 was downregulated, which interacts with members of the RAB11 protein family, leading to regulation of endocytic recycling, an important process during mycobacterial infection [49-51].

IPA was used for identification of significantly overrepresented GO categories and canonical pathways. Notably, these analyses demonstrated that the processes of cell death, apoptosis and cell survival were over-represented as GO categories and within canonical pathways levels. These pathways included: Death receptor signalling; Apoptosis signalling; TNFR2 signalling; TNFR1 signalling; PTEN signalling; and TWEAK signalling. Inspection of these pathways revealed that the genes encoding TNF- $\alpha$ and the tumour necrosis factor (ligand) superfamily, member 10 (TNFSF-10) protein are highly upregulated. These proteins can, via their respective receptors-tumour necrosis factor 
receptor superfamily member $1 \mathrm{~A}$ (TNFRSF-1A) and TNFRSF-10A/TNFRSF-10B-trigger an activation cascade through Fas (TNFRSF6)-associated via death domain (FADD) and TNFRSF1A-associated via death domain (TRADD) [both genes encoding these proteins were upregulated in the present study]. This cascade concludes with the activation of several caspases that are associated with the induction of apoptosis (upregulation of CASP7 and CASP8 was observed here), further supporting the role of apoptosis in the host response to mycobacterial infection [25].

IPA analysis also demonstrated the importance of gene products involved with immune cell communication and chemotaxis of immune cells, including the canonical pathways Communication between innate and adaptive immune cells and The role of cytokines in mediating communication between immune cells. For example, it is well established that macrophage recognition of bacterial pathogen-association molecular patterns (PAMPs) via specific Toll-like receptors (TLRs) [upregulation of TLR3; TLR5; and TLR1O and downregulation of TLR9 was observed here] leads to increased expression of a wide range of NF-kB-inducible cytokines and chemokines. In this regard, CCL4, CCL5, CXCL10 and IL8 were upregulated in the present study. Indeed, the overall pattern of immune gene expression changes observed here using RNA-seq data was consistent with previous work from our group using microarray technology [20].

\section{Antisense strand transcript expression}

The present study identified 0.4 million sequence reads that mapped to the antisense strand and it is important to note that the phenomenon of natural antisense transcription has been observed previously in mammals $[52,53]$. We identified 6,842 putative natural antisense transcripts (NATs) that were suitable for differential expression analysis of the $M$. bovis-infected MDM relative to the non-infected control MDM. This represents $26.7 \%$ of the 25,669 currently annotated B. taurus genes, which corresponds to results obtained from previous studies of antisense strand transcription on different mammals including cattle [54-58]. Of the 6,842 NATs detected, 757 were significantly differentially expressed; these comprised 558 that were upregulated and 199 that were downregulated. NATs have been shown to have varying regulatory roles in eukaryotic cells, including transcriptional and post-transcriptional control, splicing event regulation, allele-specific transcript expression, RNA editing and RNA translocation [59-64]. Further analyses identified 694 genes that were differentially expressed from both the sense and antisense strand.

IPA analysis of these 757 antisense strand transcripts revealed enrichment of genes involved in several immune processes including the inflammatory response, pattern recognition receptor signalling, cell death and apoptosis, immune cell movement and interaction, and antigen presentation. These findings suggest that NATs may play a significant role in regulating the host macrophage response following infection with pathogenic agents, including mycobacteria $[52,65]$.

\section{Comparison with microarray and real time qRT-PCR}

The MDM samples infected with $M$. bovis used for the present study had previously been analysed using the Affymetrix ${ }^{\circledR}$ GeneChip ${ }^{\circledR}$ Bovine Genome Array. Consequently, it was possible to directly compare gene expression results from microarray and sense strand RNA-seq data. The percentage of overlapping genes between the two platforms was estimated at $47.8 \%$ for the microarray and $37.3 \%$ for the RNA-seq data. Similar estimates of correspondence between RNA-seq and microarrays platforms have been reported in studies of the rat and Candida parapsilosis transcriptomes [28,66]. However, it should be noted that compared to the study of $\mathrm{Su}$ et al. [28] we observed a greater correlation between the foldchange in expression for: (1) all common genes that passed filtering detected by both platforms (6,183 genes; Spearman correlation coefficient of $0.858 ; P \leq 0.001$ ), and (2) the differentially expressed genes common to both platforms (965 genes; Spearman correlation coefficient of $0.935 ; P \leq 0.001)$.

There are a number of possible reasons for the differences observed between the two gene expression platforms used in the current study. Firstly, there are differences in the dynamic range of the two platforms: our results show that RNA-seq has a larger dynamic range and sensitivity than the microarray; similar results have recently been obtained by Chen and colleagues [27]. Secondly, the stringent expression thresholds that we have applied to the RNA-seq data in order to reduce the number of false-positive differentially expressed genes, have also reduced the number of genes common to both platforms. Thirdly, the probes on the Affymetrix ${ }^{\circledR}$ GeneChip $^{\circledR}$ Bovine Genome Array are based on sequences from the $3^{\prime}$ end of genes and are therefore $3^{\prime}$ biased [67]; RNA-seq reads are more randomly distributed across gene transcripts. Fourthly, microarrays are susceptible to cross-hybridisation, particularly among members for the same gene family that can result in elevated falsepositive rates [30-32]. Fifthly, there are differences in the statistical models used to detect differentially expressed genes for the two platforms (for example, analysis of the microarray data involve moderated $t$-tests; analysis of the RNA-seq data using edgeR involved a negative binomial distribution) [28,68-71]. Sixthly, differences in the bovine genome resources used to design the Affymetrix ${ }^{\circledR}$ GeneChip $^{\circledR}$ Bovine Genome Array (May 2005, Gene Expression Omnibus platform accession number GPL2112) 
and the B. taurus reference genome (Btau 4.0.63 genome release) used to analyse the RNA-seq data may also impact on the analysis as recently proposed in a study of the yeast transcriptome [72]. Seventhly, it has been demonstrated that increased sequencing depth also contributes to greater correspondence between RNA-seq and microarray platforms, such as that recently observed in a study of the Saccharomyces cerevisiae transcriptome [36,72].

It is important to note, however, that despite the moderate concordance in the number of differentially expressed genes common to both transcriptomic platforms, we did observe a high concordance between the GO categories and canonical pathways identified by IPA analysis. This indicates that the RNA-seq and microarray platforms both provide gene expression data that can be used for meaningful biological interpretation.

\section{Conclusions}

The results from the present study highlight the ability of the RNA-seq technologies to reveal novel features of the bovine macrophage transcriptome in response to infection with $M$. bovis, including the detection of putative NAT expression. These transcriptional signatures highlight the complex interactions between host macrophages and the mycobacterial pathogen during infection. Further analyses involving the comparison of RNA-seq -generated gene expression profiles in bovine macrophages to non-pathogenic mycobacteria, such as $M$. bovis-BCG, may enable fine-scaled interrogation of key cellular pathways that contribute to the development of pathology.

\section{Methods}

\section{MDM preparation, MDM infection and RNA purification}

The materials and methods used to isolate, purify and infect bovine MDM with $M$. bovis have been previously described in detail by us [20]. Briefly, MDM were purified from peripheral blood mononuclear cells prepared from whole blood extracted from seven age-matched (fouryears old) Holstein-Friesian females (Additional file 1: Table S1). All seven animals were selected from a herd without a recent history of bovine tuberculosis infection, which was confirmed using the single intradermal tuberculin test. MDM were cultured over an eight-day period with routine changing of culture media every two days. MDM were visually inspected using microscopy, counted and seeded in 24-well tissue culture plates at a density of $2 \times 10^{5}$ cells per well prior to infection.

For the MDM infections, $4 \times 10^{5} \mathrm{M}$. bovis cells (as determined from bacterial cell counts performed using a Petroff Hausser chamber and confirmed by colonyforming unit [cfu] counts) were added to each tissue culture plate well (MOI 2:1). The M. bovis M2137 strain bearing the SB0142 spoligotype was used for the infection experiments and non-infected control MDM received culture media only. Both non-infected control and infected MDM were prepared in adjacent duplicate tissue culture plate wells. The culture media in all wells for both non-infected control and infected MDM was replaced $2 \mathrm{~h}$ post-infection with fresh culture media and plates were then re-incubated at $37^{\circ} \mathrm{C}, 5 \% \mathrm{CO}_{2}$ until the MDM were harvested. Culturing of $M$. bovis and the MDM infections were performed in a Biosafety Containment Level 3 (CL3) laboratory.

Infected and non-infected control MDM were harvested using RLT buffer from the RNeasy Mini kit (Qiagen Ltd., Crawley, UK) supplemented with $1 \% \beta$-mercaptoethanol (Sigma-Aldrich Ireland Ltd., Dublin, Ireland) $24 \mathrm{~h}$ postinfection. For each control and treatment, MDM lysates from duplicate culture plate wells were pooled and stored at $-80^{\circ} \mathrm{C}$ until required for RNA extraction. All RNA extractions were performed in the CL3 laboratory using an RNeasy kit incorporating an on-column DNase treatment step according to the manufacturer's instructions (Qiagen). RNA quantity and quality was ascertained using a NanoDrop ${ }^{\text {тм }} 1000$ spectrophotometer (Thermo Fisher Scientific Ltd., Waltham, MA, USA) and an Agilent 2100 Bioanalyzer with the RNA 6000 Nano LabChip kit (Agilent Technologies Ltd., Cork, Ireland). All samples displayed a 260/280 ratio greater than 2.0 and RNA integrity numbers $\geq 8.5$ [73].

\section{Strand-specific RNA-seq library preparation}

In total, 14 strand-specific RNA libraries for highthroughput sequencing were prepared (seven libraries for each treatment: $M$. bovis-infected and control samples) using $200 \mathrm{ng}$ of total RNA. Total RNA was first heated at $65^{\circ} \mathrm{C}$ for $5 \mathrm{~min}$ to disrupt any secondary structure. Purification of poly(A) RNA was performed using a Dynabeads ${ }^{\circledR}$ mRNA DIRECT ${ }^{\mathrm{Tm}}$ Micro Kit according to the manufacturer's instructions (Invitrogen ${ }^{\mathrm{Tm}} / \mathrm{Life}$ Technologies Ltd., Paisley, UK). Purified poly(A) RNA was then fragmented using $1 \times$ RNA Fragmentation Reagent (Ambion ${ }^{\circledR} /$ Life Technologies Corporation, Warrington, UK) for $5 \mathrm{~min}$ at $70^{\circ} \mathrm{C}$ and precipitated using $68 \mathrm{mM}$ sodium acetate $\mathrm{pH} 5.2$ (Ambion), $227 \mathrm{ng} / \mu \mathrm{l}$ glycogen (Ambion) and $30 \mu \mathrm{l}$ of $100 \%$ ethanol (Sigma-Aldrich Ltd., Dublin, Ireland). Pellets were washed with $80 \%$ ethanol, air-dried for $10 \mathrm{~min}$ at room temperature and re-suspended in $10.5 \mu \mathrm{l}$ DNase- and RNase-free water.

Synthesis of first strand cDNA was performed by incubating fragmented RNA with $261 \mathrm{mM}$ Random Hexamer Primers (Invitrogen), $1 \times$ first strand buffer (Invitrogen); $10 \mathrm{mM}$ DTT (Invitrogen); $0.5 \mathrm{mM}$ dNTPs; $20 \mathrm{U}$ RNaseOUT $^{\mathrm{ma}}$ Recombinant Ribonuclease Inhibitor; and $200 \mathrm{U}^{\circ}$ SuperScript ${ }^{\circledR}$ II Reverse Transcriptase (Invitrogen) at $25^{\circ} \mathrm{C}$ for $10 \mathrm{~min}$, at $42^{\circ} \mathrm{C}$ for $50 \mathrm{~min}$, and $70^{\circ} \mathrm{C}$ for $15 \mathrm{~min}$. First strand synthesis reaction mixtures were 
purified using MicroSpin ${ }^{\text {Tw }}$ G-50 columns according to the manufacturer's instructions (GE Healthcare UK Ltd., Little Chalfont, Buckinghamshire, UK).

Second strand cDNA synthesis, involving the incorporation of uracil, was performed by adding the first strand cDNA synthesis reaction to a second strand reaction mix consisting of $0.065 \times$ first strand buffer (Invitrogen); $1 \times$ second strand buffer (Invitrogen); a dNTP mix consisting of a final concentration of $0.3 \mathrm{mM}$ dATP, dCTP, dGTP (Sigma-Aldrich) and $0.3 \mathrm{mM}$ dUTP (Bioline Reagents Ltd., London, UK); 1 mM DTT (Invitrogen); 2 U RNase H (Invitrogen) and $50 \mathrm{U} \quad$ E. coli DNA Polymerase I (Invitrogen). Reactions were incubated at $16^{\circ} \mathrm{C}$ for $2.5 \mathrm{~h}$. The double stranded cDNA was subsequently purified using a QIAquick PCR Purification kit (Qiagen) according to the manufacturer's instructions and eluted in $30 \mu \mathrm{l}$ of the provided elution buffer.

Blunt-end repair of cDNA was performed in a $100 \mu \mathrm{l}$ reaction containing $1 \times$ T4 DNA ligase buffer with $10 \mathrm{mM}$ dATP (New England Biolabs ${ }^{\circledR}$ Inc., MA, USA), $0.4 \mathrm{mM}$ of each dNTP (Invitrogen), $15 \mathrm{U}$ T4 DNA polymerase (New England Biolabs), 5 U DNA Polymerase I Large [Klenow] Fragment (New England Biolabs) and $50 \mathrm{U}$ T4 polynucleotide kinase (New England Biolabs). Reactions were incubated at $20^{\circ} \mathrm{C}$ for $30 \mathrm{~min}$ and the cDNA was then purified using a QIAquick PCR Purification Kit (Qiagen) according to the manufacturer's instructions and eluted in $32 \mu \mathrm{l}$ of the provided elution buffer.

To facilitate Illumina ${ }^{\circledR} \mathrm{GA}$ adaptor ligation, a single ' $\mathrm{A}$ ' base was added to the $3^{\prime}$ ends of the blunt-end repaired cDNA samples. $32 \mu \mathrm{l}$ of purified phosphorylated blunt end-repaired cDNA was included in a final $50 \mu \mathrm{l}$ reaction mixture containing: $1 \times$ Klenow fragment buffer (New England Biolabs); $0.2 \mathrm{mM}$ dATP (Invitrogen), and $15 \mathrm{U}$ Klenow fragment with $3^{\prime}$-to- $5^{\prime}$ exonuclease activity (New England Biolabs). Reactions were incubated at $37^{\circ} \mathrm{C}$ for $30 \mathrm{~min}$, after which cDNA was purified using a QIAquick MinElute Kit (Qiagen) according to the manufacturer's instructions and eluted in $21 \mu \mathrm{l}$ of the provided elution buffer.

Illumina ${ }^{\circledR}$ RNA-seq adaptor ligation reactions $(50 \mu \mathrm{l}$ volumes) involved incubation of $21 \mu \mathrm{l}$ of phosphorylated blunt-ended cDNA containing a 3'-dATP overhang with 1× Quick DNA ligase buffer (New England Biolabs); 30 $\mathrm{nM}$ custom indexed single-read adaptors [Additional file 1: Table S1] and 15 U T4 DNA ligase (Invitrogen). Reaction mixes were incubated at room temperature for $15 \mathrm{~min}$ and purified using a QIAquick MinElute Kit according to the manufacturer's instructions (Qiagen) and eluted in $10 \mu \mathrm{l}$ of the provided elution buffer. Adaptor-ligated cDNA was gel-purified using 2.5\% agarose gels stained with $1 \mu \mathrm{g} / \mathrm{ml}$ ethidium bromide (Invitrogen). Gels were electrophoresed at 100 Volts using $1 \times$ TAE buffer (Invitrogen) for $75 \mathrm{~min}$ at room temperature. Size fractionated bands corresponding to $200 \mathrm{bp}(+50 \mathrm{bp})$ were excised from each sample and purified using a QIAquick Gel Extraction kit (Qiagen) according to the manufacturer's instructions and eluted in $30 \mu \mathrm{l}$ of elution buffer.

To generate strand-specific RNA-seq libraries, the second strand of the gel-purified adapter-ligated cDNA containing uracil was digested enzymatically in $30 \mu \mathrm{l}$ reaction volumes containing $1 \times$ Uracil-DNA Glycosylase buffer and 1 U Uracil-DNA Glycosylase (Bioline). Reactions were incubated at $37^{\circ} \mathrm{C}$ for $15 \mathrm{~min}$ followed by $94^{\circ} \mathrm{C}$ for $10 \mathrm{~min}$.

PCR enrichment amplifications (50 $\mu \mathrm{l})$ containing $15 \mu \mathrm{l}$ of second strand-digested, adaptor-ligated cDNA; $1 \times$ Phusion ${ }^{\circledR}$ High-Fidelity DNA polymerase buffer (New England Biolabs); $334 \mathrm{nM}$ each Illumina ${ }^{\circledR}$ PCR primer (Illumina ${ }^{\circledR}$ Inc., San Diego, CA, USA); $0.4 \mathrm{mM}$ each of dATP, dCTP, DGTP and dTTP (Invitrogen) and $1 \mathrm{U}$ Phusion ${ }^{\circledR}$ High-Fidelity DNA polymerase (New England Biolabs). PCR amplification reactions consisted of an initial denaturation step of $98^{\circ} \mathrm{C}$ for 30 seconds, 18 cycles of $98^{\circ} \mathrm{C}$ for 10 seconds, $65^{\circ} \mathrm{C}$ for 30 seconds and $72^{\circ} \mathrm{C}$ for 30 seconds, followed by a final extension step of $72^{\circ} \mathrm{C}$ for 5 min. PCR products were visualised following electrophoresis on a $2 \%$ agarose gel stained with ethidium bromide $(0.6 \mu \mathrm{g} / \mathrm{ml}$; Invitrogen $)$ and purified to remove PCRgenerated adaptor-dimers using an Agencourt AMPure XP kit (Beckman Coulter Genomics, Danvers, MA, USA) according to the manufacturer's instructions with final elution in $30 \mu \mathrm{l}$ of $1 \times \mathrm{TE}$ buffer.

All RNA-seq libraries were quantified using a Qubit $^{\circledR}$ Fluorometer and Qubit ${ }^{\circledR}$ double stranded DNA High Sensitivity Assay Kit (Invitrogen). RNA-seq library quality was assessed using an Agilent Bioanalyzer and Agilent High sensitivity DNA chip (Agilent) and confirmed that library insert sizes were $\sim 200-250$ bp for all individual libraries. Individual RNA-seq libraries were standardised and pooled in equimolar quantities $(10 \mu \mathrm{M}$ for each individual library). The quantity and quality of the final pooled library was assessed as described above prior to sequencing.

Cluster generation and sequencing of the pooled RNAseq library was carried out on an Illumina ${ }^{\circledR}$ Cluster Station and Illumina ${ }^{\circledR}$ Genome Analyzer IIx sequencer according to the manufacturer's instructions (Illumina). The pooled library was sequenced as single-end read 84mers using Illumina ${ }^{\circledR}$ version 4.0 sequencing kits and the standard Illumina ${ }^{\circledR}$ Genome Analyzer $\mathrm{II}_{\mathrm{x}}$ pipeline. The Illumina ${ }^{\circledR}$ Sequencing Control Software version 2.9 and Real Time Analysis version 1.9 software packages were used for real-time tracking of the sequencing run, realtime image processing, the generation of base intensity values and base calling. These RNA-seq data have been deposited in the NCBI Gene Expression Omnibus (GEO) database with experiment series accession number GSE45439. 


\section{Statistical analysis of RNA-seq data}

Sequence reads obtained from seven lanes of the Illumina flow cell were deconvoluted into 14 individual libraries using the unique indexed barcoded adapters. A Perl script was used to screen adapter artefacts, removing any reads containing a full-length match to the 33 nucleotide Illumina ${ }^{\circledR}$ adapter sequence, allowing up to four mismatches. Read quality was then assessed using FastQC software [version 0.9] (www.bioinformatics. bbsrc.ac.uk/projects/fastqc), revealing low Phred scores at the $3^{\prime}$ end of sequence reads. Consequently, nine nucleotides were trimmed from the $3^{\prime}$ ends of all sequence reads using the FASTX Toolkit [version 0.0.13] (http:// hannonlab.cshl.edu/fastx_toolkit) generating usable reads of 69 nucleotides.

Deconvoluted quality-checked sequence reads were aligned to the B. taurus reference genome (Btau 4.0.63 genome release) with the TopHat splice junction mapper [version 1.3.0] [74,75], which aligns reads using the Bowtie aligner (version 0.12.7). The Bowtie alignment procedure was configured for strand-specific libraries and to filter only unique hits to the reference genome sequence. In addition, all sequence reads were aligned to the $M$. bovis AF2122/97 chromosome, complete genome (accession number NC_002945.3) as detailed above. To obtain raw counts per transcript, HTSeq package [version 0.5.3p1] (http://www-huber.embl.de/users/anders/ HTSeq/doc/overview.html) was used on alignment files in BAM format. HTSeq-count was used with option overlap resolution mode set to intersection non-empty. Counts of uniquely-mapped reads were obtained for all bovine Ensembl genes and transcripts, with separate counts obtained for sense and antisense DNA strands.

Once raw counts were obtained from HTseq-count, analysis of differential expression was performed in the $R$ statistical programming environment [76] using the edgeR (version 2.2.5) Bioconductor package [70,77] and lattice [version 0.19-30] (http://lattice.r-forge.r-project. org). First, quality checks were performed by plotting the density of counts per feature (a feature being a gene or transcript generated respectively from the sense or antisense strand) for each sample and also by generating a multidimensional scaling plot of the RNA-seq data. The edgeR software package was used to determine differential expression using the paired-sample statistical test. Filtering of lowly expressed features was performed by retaining only features with at least one count per million in three or more libraries. A normalisation factor was calculated using the default trimmed mean of $M$ values (TMM) method [70,77], and the dispersion parameter for each feature was estimated as the Cox-Reid common dispersion method in the edgeR package. Differential expression was evaluated by fitting a negative binomial generalized linear model for each feature and then adjusting the $P$-value for multiple testing using the Benjamini-Hochberg correction [78] with a false discovery rate (FDR) of 0.05 .

For RNA-seq dynamic range analysis we used two different normalisation strategies: (1) RPKM values generated by the edgeR package; and (2) quantile normalisation of RNA-seq data performed using the Linear Models for Microarray Data (LIMMA) package [71]. RPKM and quantile-normalised counts were not used for differential expression analysis.

\section{Reanalysis and reannotation of Affymetrix ${ }^{\circledR}$ GeneChip ${ }^{\circledR}$ Bovine Genome Array data}

The RNA samples analysed in the current study were previously analysed by us using the Affymetrix ${ }^{\circledR}$ GeneChip ${ }^{\circledR}$ Bovine Genome Array [20]. These microarray data have been deposited in the NCBI GEO database with experiment series accession number GSE33309. In order to compare gene expression profiles from these samples using RNA-seq and microarray technologies, we first reanalysed the microarray data using a series of Bioconductor packages.

Firstly, quality control analysis was performed on all microarrays using the Simpleaffy software package [79]. Then the raw microarray expression values were normalised using the gcRMA package [80]. The raw data followed an additional normalisation step using the Factor Analysis for Robust Microarray Summarization (FARMS) algorithm to remove probe sets with high noise:signal ratios [81], these normalised data were then further subjected to filtering for informative probe sets using informative/non-informative calls (I/NI-calls) package [82]. The obtained I/NI filtering list was then applied to the gcRMA normalised data to remove all noninformative probes. Differentially expressed genes were identified from the filtered gcRMA normalised data using the LIMMA package. The Benjamini-Hochberg multiple testing correction method [78] was applied to all differentially expressed genes to minimise the FDR and adjusted $P$-values for differentially expressed genes were calculated. Probe sets displaying differential expression between control and infected samples were annotated to Ensembl gene IDs using biomaRt package [83].

Supplementary statistical analyses were performed using the SPSS software package (version 20; www-01. ibm.com/software/analytics/spss).

\section{IPA analyses}

Ingenuity ${ }^{\circledR}$ Systems Pathway Analysis (IPA, Ingenuity Systems, Redwood City, CA, USA; release date November 2012) was used to identify canonical pathways and functional processes of biological importance within the list of differentially expressed genes identified with RNA-seq and microarray platforms. The Ingenuity ${ }^{\circledR}$ Knowledge Base 
contains the largest database of manually-curated and experimentally-validated physical, transcriptional and enzymatic molecular interactions. Furthermore, each interaction in the Ingenuity ${ }^{\circledR}$ Knowledge Base is supported by previously published information.

Functional analysis of genes was performed using IPA to characterise the GO categories of differentially expressed genes between the control and M. bovis-infected MDM. For this, IPA performed an over-representation analysis that categorises differentially expressed genes into functional groups using the Ingenuity ${ }^{\circledR}$ Knowledge Base. Each category in IPA is ranked based on the number of differentially expressed genes in each functional group. The right-tailed Fisher's exact test was used to calculate a $P$ value for each GO category assigned to differentially expressed genes.

Ingenuity ${ }^{\circledR}$ Systems Pathway Analysis contains a large library of known canonical pathways that were overlaid with the differentially expressed genes to identify major biological pathways associated with $M$. bovis infection in MDM. The significance of the association between differentially expressed genes and each canonical pathway was assessed using two methods. Firstly, a ratio was estimated from the number of molecules from the differentially expressed gene data set that map to each pathway, compared to the total number of molecules that map to the canonical pathway based on the reference gene list; and secondly, a Fisher's exact test that generates a $P$-value for the assignment of the differentially expressed genes to a particular canonical pathway compared to the reference gene list. Canonical pathways were then overlaid with the expression values of the differentially expressed genes.

\section{Additional files}

Additional file 1: Table S1. RNA-seq libraries information. The indexed adapter sequence, animal sample ID, sample treatment, pooling strategy, and sequencing read information (number and percentage) before and after data filtering are given for each RNA-seq library.

Additional file 2: Figure S1. Density plot of the distribution of reads per gene. Density plots of the number of sequence reads (in $\log _{10}$ space) per gene for each RNA-seq library sample.

Additional file 3: Table S2. The list of all significant differentially expressed genes detected following M. bovis infection based on RNA-seq sense strand data. For each differentially expressed gene is shown its gene name, $\log _{2}$ fold-change, $P$-value, adjusted $P$-value (BenjaminiHochberg correction), description and Ensembl gene ID. The biomaRt package and the $B$. taurus reference genome were used to obtain gene names and gene descriptions. Genes without names or descriptions are stated here as "not available".

Additional file 4: Table S3. GO categories identified using IPA based on RNA-seq sense strand data. The top ranking GO categories identified by IPA based on RNA-seq sense strand data are ranked according to $P$ values.

Additional file 5: Table S4. Significant canonical pathways identified using IPA based on RNA-seq sense strand data. The canonical pathways identified by IPA based on RNA-seq sense strand data are ranked according to $P$-values. The ratio indicates the number of differentially expressed genes involved in each canonical pathway divided by the total number of genes/molecules within each pathway according to the IPA Knowledge Base.

Additional file 6: Table S5. The list of all significant differentially expressed genes detected following $M$. bovis infection based on RNA-seq antisense strand data. For each differentially expressed gene is shown its gene name, $\log _{2}$ fold-change, $P$-value, adjusted $P$-value (BenjaminiHochberg correction), description and Ensembl gene ID. The biomaRt package and the $B$. taurus reference genome were used to obtain gene names and gene descriptions. Genes without names or descriptions are stated here as "not available".

Additional file 7: Figure S2. Integrative Genomics Viewer (IGV) screen capture of reads mapping to SPTB gene. This figure shows the distribution of sense (represented in red) and antisense (represented in blue) strand reads that mapped to the 31 end of spectrin, beta, erythrocytic gene (SPTB).

Additional file 8: Table S6. GO categories identified using IPA based on RNA-seq antisense strand data. The top ranking GO categories identified by IPA based on RNA-seq antisense strand data are ranked according to $P$-values.

Additional file 9: Table S7. Significant canonical pathways identified using IPA based on RNA-seq antisense strand data. The canonical pathways identified by IPA based on RNA-seq antisense strand data are ranked according to $P$-values. The ratio indicates the number of differentially expressed genes involved in each canonical pathway divided by the total number of genes/molecules within each pathway according to the IPA ${ }^{\circledR}$ Knowledge Base

Additional file 10: Figure S3. Multi-dimensional scale plot of all M. bovis-infected and control samples based on Microarray data. Dimension 1 and dimension 2 separate all 12 samples based on the expression value of the 11,790 probes (based on microarray data only) that passed all data filtering criteria prior to differential gene expression analysis.

Additional file 11: Table S8. The list of all significant differentially expressed genes detected following $M$. bovis infection based on microarray data. For each differentially expressed gene is shown its gene name, $\log _{2}$ fold-change, $P$-value, adjusted $P$-value (Benjamini-Hochberg correction), description and Ensembl gene ID. The biomaRt package and the $B$. taurus reference genome were used to obtain gene names and gene descriptions. Genes without names or descriptions are stated here as "not available".

Additional file 12: Table S9. List of all significant differentially expressed genes detected following $M$. bovis infection common to both microarray and RNA-seq sense strand data. For each differentially expressed gene is shown its Ensembl gene ID, gene name, description, $\log _{2}$ fold-change, $P$-value and adjusted $P$-value (Benjamini-Hochberg correction) based on microarray and RNA-seq sense strand data. The biomaRt package and the $B$. taurus reference genome were used to obtain gene names and gene descriptions. Genes without names or descriptions are stated here as "not available".

Additional file 13: Figure S4. Density plots of $\log _{2}$ mean CPM and $\log _{2}$ mean hybridisation intensities for differentially expressed genes unique and common to both platforms. This analysis was performed for each platform/treatment group. DEG, differentially expressed genes.

Additional file 14: Table S10. GO categories identified using IPA based on microarray data. The top ranking GO categories identified by IPA based on microarray data are ranked according to $P$-values.

Additional file 15: Table S11. GO categories identified using IPA based on differentially expressed genes common to both microarray and RNAseq sense strand data. The top ranking GO categories identified by IPA based on differentially expressed genes common to both microarray and RNA-seq sense strand data are ranked according to $P$-values.

Additional file 16: Table S12. Significant canonical pathways identified using IPA based on microarray data. The canonical pathways identified by IPA based on microarray data are ranked according to $P$-values. The ratio indicates the number of differentially expressed genes involved in each canonical pathway divided by the total number of genes/molecules within each pathway according to the IPA ${ }^{\circledR}$ Knowledge Base. 
Additional file 17: Table S13. Significant canonical pathways identified using IPA based on differentially expressed genes common to both microarray and RNA-seq sense strand data. The canonical pathways identified by IPA based on differentially expressed genes common to both microarray and RNA-seq sense strand data are ranked according to $P$-values. The ratio indicates the number of differentially expressed genes involved in each canonical pathway divided by the total number of genes/molecules within each pathway according to the IPA ${ }^{\circledR}$ Knowledge Base.

\section{Competing interests}

The authors declare that they have no competing interests.

\section{Authors' contributions}

Conceived and designed the experiments: NCN, DAM, MT, JAB, AJL, BJL, EG, SVG, DEM. Performed the experiments: NCN, DAM, MT, JAB, KMC. Analysed the data: NCN, SDEP, DAM, MT, KRA, KEK, KH, JAB. Prepared and edited the manuscript: NCN, DAM, SDEP, MT, KRA, KEK, KH, AJL, BJL, EG, SVG, DEM. All authors read and approved the manuscript.

\section{Acknowledgements}

We would like to thank Mr. Eamon Costello and staff at the Irish Department of Agriculture, Food and the Marine Backweston Laboratory Campus (Celbridge, Co. Kildare) for providing the isolate of M. bovis. We also would like to thank the staff at the UCD Lyons Research Farm for assistance with blood collection. We also thank Dr. Paul McGettigan of the UCD Reproductive Biology Research Cluster and the members of the UCD Tuberculosis Diagnostics and Immunology Research Centre. Finally, we thank two anonymous reviewers for helpful comments and insights. This work was funded by the following sources: Science Foundation Ireland (www.sfi.ie) Investigator grants (Nos: SFI/01/F.1/B028 and SFI/08/IN.1/B2038); Department of Agriculture, Fisheries and Food (www.agriculture.gov.ie) Research Stimulus Grant (No: RSF 06 405); European Union Framework 7 (http://cordis.europa. eu/fp7) Project Grant (No: KBBE-211602-MACROSYS); Irish Research Council for Science, Engineering and Technology (IRCSET) funded Bioinformatics and Systems Biology PhD Programme (http://bioinfo-casl.ucd.ie/PhD). The funders had no role in study design, data collection and analysis, decision to publish, or preparation of the manuscript.

\section{Author details}

${ }^{1}$ Animal Genomics Laboratory, UCD School of Agriculture and Food Science, University College Dublin, Belfield, Dublin 4, Dublin, Ireland. ${ }^{2}$ UCD School of Veterinary Medicine, University College Dublin, Belfield, Dublin 4, Dublin, Ireland. ${ }^{3}$ Smurfit Institute of Genetics, Trinity College Dublin, Trinity College, Dublin, Ireland. ${ }^{4}$ UCD Conway Institute of Biomolecular and Biomedical Research, University College Dublin, Belfield, Dublin 4, Dublin, Ireland. ${ }^{5}$ Tuberculosis Diagnostics and Immunology Research Centre, UCD School of Veterinary Medicine, University College Dublin, Belfield, Dublin 4, Dublin, Ireland.

Received: 30 August 2012 Accepted: 8 March 2013

Published: 8 April 2013

\section{References}

1. Brosch R, Gordon SV, Marmiesse M, Brodin P, Buchrieser C, Eiglmeier K, Garnier T, Gutierrez C, Hewinson G, Kremer K, et al: A new evolutionary scenario for the Mycobacterium tuberculosis complex. Proc Natl Acad Sci USA 2002, 99(6):3684-3689.

2. Djelouadji Z, Raoult D, Drancourt M: Palaeogenomics of Mycobacterium tuberculosis: epidemic bursts with a degrading genome. Lancet Infect Dis 2011, 11(8):641-650.

3. Wirth T, Hildebrand F, Allix-Beguec C, Wolbeling F, Kubica T, Kremer K, van Soolingen D, Rusch-Gerdes S, Locht C, Brisse S, et al: Origin, spread and demography of the Mycobacterium tuberculosis complex. PLoS Pathog 2008, 4(9):e1000160.

4. Smith NH, Gordon SV, de la Rua-Domenech R, Clifton-Hadley RS, Hewinson RG: Bottlenecks and broomsticks: the molecular evolution of Mycobacterium bovis. Nat Rev Microbiol 2006, 4(9):670-681.

5. Good M: Bovine tuberculosis eradication in Ireland. Irish Veterinary Journal 2006, 59(3):154-162
6. More SJ, Good M: The tuberculosis eradication programme in Ireland: a review of scientific and policy advances since 1988. Vet Microbiol 2006, 112(2-4):239-251.

7. Pollock JM, Neill SD: Mycobacterium bovis infection and tuberculosis in cattle. Vet J 2002, 163(2):115-127

8. Harding CV, Boom WH: Regulation of antigen presentation by Mycobacterium tuberculosis: a role for Toll-like receptors. Nat Rev Microbiol 2010, 8(4):296-307.

9. Kunnath-Velayudhan S, Gennaro ML: Immunodiagnosis of tuberculosis: a dynamic view of biomarker discovery. Clin Microbiol Rev 2011, 24(4):792-805.

10. Pollock JM, Welsh MD, McNair J: Immune responses in bovine tuberculosis: towards new strategies for the diagnosis and control of disease. Vet Immunol Immunopathol 2005, 108(1-2):37-43.

11. Waters WR, Palmer MV, Thacker TC, Davis WC, Sreevatsan S, Coussens $P$, Meade KG, Hope JC, Estes DM: Tuberculosis immunity: opportunities from studies with cattle. Clin Dev Immunol 2011, 2011:768542.

12. Sundaramurthy $V$, Pieters J: Interactions of pathogenic mycobacteria with host macrophages. Microbes Infect 2007, 9(14-15):1671-1679.

13. Meena LS, Rajni: Survival mechanisms of pathogenic Mycobacterium tuberculosis H37Rv. FEBS J 2010, 277(11):2416-2427.

14. Walzl G, Ronacher K, Hanekom W, Scriba TJ, Zumla A: Immunological biomarkers of tuberculosis. Nat Rev Immunol 2011, 11(5):343-354

15. MacHugh DE, Gormley E, Park SD, Browne JA, Taraktsoglou M, O'Farrelly C, Meade KG: Gene expression profiling of the host response to Mycobacterium bovis infection in cattle. Transbound Emerg Dis 2009, 56(6-7):204-214.

16. Widdison S, Watson M, Coffey TJ: Early response of bovine alveolar macrophages to infection with live and heat-killed Mycobacterium bovis. Dev Comp Immunol 2011, 35(5):580-591.

17. Meade KG, Gormley E, Doyle MB, Fitzsimons T, O'Farrelly C, Costello E, Keane J, Zhao Y, MacHugh DE: Innate gene repression associated with Mycobacterium bovis infection in cattle: toward a gene signature of disease. BMC Genomics 2007, 8:400.

18. Kabara E, Kloss CC, Wilson M, Tempelman RJ, Sreevatsan S, Janagama H, Coussens PM: A large-scale study of differential gene expression in monocyte-derived macrophages infected with several strains of Mycobacterium avium subspecies paratuberculosis. Brief Funct Genomics 2010, 9(3):220-237.

19. MacHugh D, Taraktsoglou M, Killick K, Nalpas N, Browne J, Park S, Hokamp K, Gormley E, Magee D: Pan-genomic analysis of bovine monocytederived macrophage gene expression in response to in vitro infection with Mycobacterium avium subspecies paratuberculosis. Vet Res 2012, 43(1):25

20. Magee DA, Taraktsoglou M, Killick KE, Nalpas NC, Browne JA, Park SDE, Conlon KM, Lynn DJ, Hokamp K, Gordon SV, et al: Global gene expression and systems biology analysis of bovine monocyte-derived macrophages in response to in vitro challenge with Mycobacterium bovis. PLoS One 2012, 7(2):e32034.

21. Killick KE, Browne JA, Park SD, Magee DA, Martin I, Meade KG, Gordon SV, Gormley E, O'Farrelly C, Hokamp K, et al: Genome-wide transcriptional profiling of peripheral blood leukocytes from cattle infected with Mycobacterium bovis reveals suppression of host immune genes. BMC Genomics 2011, 12(1):611.

22. Wilhelm BT, Landry JR: RNA-Seq-quantitative measurement of expression through massively parallel RNA-sequencing. Methods 2009, 48(3):249-257.

23. Ozsolak F, Milos PM: RNA sequencing: advances, challenges and opportunities. Nat Rev Genet 2011, 12(2):87-98.

24. Roy NC, Altermann E, Park ZA, McNabb WC: A comparison of analog and next-generation transcriptomic tools for mammalian studies. Brief Funct Genomics 2011, 10(3):135-150.

25. Behar SM, Martin CJ, Booty MG, Nishimura T, Zhao X, Gan HX, Divangahi M, Remold HG: Apoptosis is an innate defense function of macrophages against Mycobacterium tuberculosis. Mucosal Immunol 2011, 4(3):279-287.

26. GeneChip ${ }^{\circledR}$ Bovine Genome Array data sheet. http://media.affymetrix.com/ support/technical/datasheets/bovine_datasheet.pdf.

27. Chen H, Liu Z, Gong S, Wu X, Taylor WL, Williams RW, Matta SG, Sharp BM: Genome-wide gene expression profiling of nucleus accumbens neurons projecting to ventral pallidum using both microarray and transcriptome sequencing. Frontiers in neuroscience 2011, 5:98.

28. Su Z, Li Z, Chen T, Li QZ, Fang H, Ding D, Ge W, Ning B, Hong H, Perkins $\mathrm{RG}$, et al: Comparing next-generation sequencing and microarray 
technologies in a toxicological study of the effects of aristolochic acid on rat kidneys. Chem Res Toxicol 2011, 24(9):1486-1493.

29. Lesho E, Forestiero FJ, Hirata MH, Hirata RD, Cecon L, Melo FF, Paik SH, Murata Y, Ferguson EW, Wang Z, et al: Transcriptional responses of host peripheral blood cells to tuberculosis infection. Tuberculosis (Edinb) 2011, 91(5):390-399.

30. Bradford JR, Hey Y, Yates T, Li Y, Pepper SD, Miller CJ: A comparison of massively parallel nucleotide sequencing with oligonucleotide microarrays for global transcription profiling. BMC Genomics 2010, 11:282

31. Liu S, Lin L, Jiang P, Wang D, Xing Y: A comparison of RNA-Seq and highdensity exon array for detecting differential gene expression between closely related species. Nucleic Acids Res 2011, 39(2):578-588,

32. Marioni JC, Mason CE, Mane SM, Stephens M, Gilad Y: RNA-seq: an assessment of technical reproducibility and comparison with gene expression arrays. Genome Res 2008, 18(9):1509-1517.

33. Grabherr MG, Haas BJ, Yassour M, Levin JZ, Thompson DA, Amit I, Adiconis $X$, Fan L, Raychowdhury R, Zeng Q, et al: Full-length transcriptome assembly from RNA-Seq data without a reference genome. Nat Biotech 2011, 29(7):644-652.

34. Garber M, Grabherr MG, Guttman M, Trapnell C: Computational methods for transcriptome annotation and quantification using RNA-seq. Nat Meth 2011, 8(6):469-477.

35. Toung JM, Morley M, Li M, Cheung VG: RNA-sequence analysis of human B-cells. Genome Res 2011, 21(6):991-998.

36. Tarazona S, Garcia-Alcalde F, Dopazo J, Ferrer A, Conesa A: Differential expression in RNA-seq: a matter of depth. Genome Res 2011, 21(12):2213-2223.

37. Wang $Y$, Ghaffari N, Johnson CD, Braga-Neto UM, Wang H, Chen R, Zhou H: Evaluation of the coverage and depth of transcriptome by RNA-Seq in chickens. BMC Bioinformatics 2011, 12(Suppl 10):S5.

38. Blanco FC, Soria M, Bianco MV, Bigi F: Transcriptional response of peripheral blood mononuclear cells from cattle infected with Mycobacterium bovis. PLoS One 2012, 7(7):e41066.

39. Mirrakhimov AE, Kerimkulova AS, Lunegova OS, Moldokeeva CB, Zalesskaya YV, Abilova SS, Sovhozova NA, Aldashev AA, Mirrakhimov EM: An association between TRP64ARG polymorphism of the B3 adrenoreceptor gene and some metabolic disturbances. Cardiovasc Diabetol 2011, 10:89.

40. Lirussi F, Rakotoniaina Z, Madani S, Goirand F, Breuiller-Fouche M, Leroy MJ, Sagot P, Morrison JJ, Dumas M, Bardou M: ADRB3 adrenergic receptor is a key regulator of human myometrial apoptosis and inflammation during chorioamnionitis. Biol Reprod 2008, 78(3):497-505.

41. Yu JJ, Gaffen SL: Interleukin-17: a novel inflammatory cytokine that bridges innate and adaptive immunity. Front Biosci 2008, 13:170-177.

42. Molenaar AJ, Harris DP, Rajan GH, Pearson ML, Callaghan MR, Sommer L, Farr VC, Oden KE, Miles MC, Petrova RS, et al: The acute-phase protein serum amyloid $\mathrm{A} 3$ is expressed in the bovine mammary gland and plays a role in host defence. Biomarkers 2009, 14(1):26-37.

43. Bettler B, Kaupmann K, Mosbacher J, Gassmann M: Molecular structure and physiological functions of GABA(B) receptors. Physiol Rev 2004, 84(3):835-867.

44. Chapman RW, Hey JA, Rizzo CA, Bolser DC: GABAB receptors in the lung Trends Pharmacol Sci 1993, 14(1):26-29.

45. Dicpinigaitis PV, Dobkin JB, Rauf K , Aldrich TK: Inhibition of capsaicininduced cough by the gamma-aminobutyric acid agonist baclofen. $J$ Clin Pharmacol 1998, 38(4):364-367.

46. Halbleib JM, Nelson WJ: Cadherins in development: cell adhesion, sorting, and tissue morphogenesis. Genes Dev 2006, 20(23):3199-3214.

47. Kadarmideen HN, Ali AA, Thomson PC, Muller B, Zinsstag J: Polymorphisms of the SLC11A1 gene and resistance to bovine tuberculosis in African Zebu cattle. Anim Genet 2011, 42(6):656-658.

48. Somia NV, Schmitt MJ, Vetter DE, Van Antwerp D, Heinemann SF, Verma IM: LFG: an anti-apoptotic gene that provides protection from Fas-mediated cell death. Proc Natl Acad Sci USA 1999, 96(22):12667-12672.

49. Roberts RC, Peden AA, Buss F, Bright NA, Latouche M, Reilly MM, KendrickJones J, Luzio JP: Mistargeting of SH3TC2 away from the recycling endosome causes Charcot-Marie-Tooth disease type 4C. Hum Mol Genet 2010, 19(6):1009-1018.

50. Halaas O, Steigedal M, Haug M, Awuh JA, Ryan L, Brech A, Sato S, Husebye H, Cangelosi GA, Akira S, et al: Intracellular Mycobacterium avium intersect transferrin in the Rab11(+) recycling endocytic pathway and avoid lipocalin 2 trafficking to the lysosomal pathway. J Infect Dis 2010, 201(5):783-792.

51. Stendel C, Roos A, Kleine H, Arnaud E, Ozcelik M, Sidiropoulos PN, Zenker J, Schupfer F, Lehmann U, Sobota RM, et al: SH3TC2, a protein mutant in Charcot-Marie-Tooth neuropathy, links peripheral nerve myelination to endosomal recycling. Brain 2010, 133(Pt 8):2462-2474.

52. Wang F, Hu S, Liu W, Qiao Z, Gao Y, Bu Z: Deep-sequencing analysis of the mouse transcriptome response to infection with Brucella melitensis strains of differing virulence. PLoS One 2011, 6(12):e28485.

53. 't Hoen PA, Ariyurek Y, Thygesen HH, Vreugdenhil E, Vossen RH, de Menezes RX, Boer JM, van Ommen GJ, den Dunnen JT: Deep sequencing-based expression analysis shows major advances in robustness, resolution and inter-lab portability over five microarray platforms. Nucleic Acids Res 2008, 36(21):e141.

54. Grigoriadis A, Oliver GR, Tanney A, Kendrick H, Smalley MJ, Jat P, Neville AM: Identification of differentially expressed sense and antisense transcript pairs in breast epithelial tissues. BMC Genomics 2009, 10:324.

55. Yelin R, Dahary D, Sorek R, Levanon EY, Goldstein O, Shoshan A, Diber A, Biton S, Tamir Y, Khosravi R, et al: Widespread occurrence of antisense transcription in the human genome. Nat Biotechnol 2003, 21(4):379-386.

56. Galante PA, Vidal DO, de Souza JE, Camargo AA, de Souza SJ: Senseantisense pairs in mammals: functional and evolutionary considerations. Genome Biol 2007, 8(3):R40.

57. Zhang Y, Liu XS, Liu QR, Wei L: Genome-wide in silico identification and analysis of cis natural antisense transcripts (cis-NATs) in ten species. Nucleic Acids Res 2006, 34(12):3465-3475.

58. Katayama S, Tomaru Y, Kasukawa T, Waki K, Nakanishi M, Nakamura M, Nishida H, Yap CC, Suzuki M, Kawai J, et al: Antisense transcription in the mammalian transcriptome. Science 2005, 309(5740):1564-1566.

59. Werner A, Carlile M, Swan D: What do natural antisense transcripts regulate? RNA Biol 2009, 6(1):43-48.

60. Werner A, Sayer JA: Naturally occurring antisense RNA: function and mechanisms of action. Curr Opin Nephrol Hypertens 2009, 18(4):343-349.

61. Werner A, Swan D: What are natural antisense transcripts good for? Biochem Soc Trans 2010, 38(4):1144-1149.

62. Lapidot M, Pilpel Y: Genome-wide natural antisense transcription: coupling its regulation to its different regulatory mechanisms. EMBO Rep 2006, 7(12):1216-1222.

63. Lasa I, Toledo-Arana A, Dobin A, Villanueva M, de los Mozos IR, VergaraIrigaray M, Segura V, Fagegaltier D, Penades JR, Valle J, et al: Genome-wide antisense transcription drives mRNA processing in bacteria. Proc Natl Acad Sci USA 2011, 108(50):20172-20177.

64. Faghihi MA, Wahlestedt C: Regulatory roles of natural antisense transcripts. Nat Rev Mol Cell Biol 2009, 10(9):637-643.

65. Esterhuyse MM, Linhart HG, Kaufmann SH: Can the battle against tuberculosis gain from epigenetic research? Trends Microbiol 2012, 20(5):220-226.

66. Guida A, Lindstadt C, Maguire SL, Ding C, Higgins DG, Corton NJ, Berriman M, Butler G: Using RNA-seq to determine the transcriptional landscape and the hypoxic response of the pathogenic yeast Candida parapsilosis. BMC Genomics 2011, 12:628.

67. Fasold M, Binder H: Estimating RNA-quality using GeneChip microarrays. BMC Genomics 2012, 13(1):186.

68. Fang Z, Martin JA, Wang Z: Statistical methods for identifying differentially expressed genes in RNA-Seq experiments. Cell \& bioscience 2012, 2(1):26

69. Mortazavi A, Williams BA, McCue K, Schaeffer L, Wold B: Mapping and quantifying mammalian transcriptomes by RNA-Seq. Nat Methods 2008, 5(7):621-628.

70. Robinson MD, McCarthy DJ, Smyth GK: edgeR: a Bioconductor package for differential expression analysis of digital gene expression data. Bioinformatics 2010, 26(1):139-140.

71. Smyth GK: Linear models and empirical bayes methods for assessing differential expression in microarray experiments. Stat Appl Genet Mol Biol 2004, 3:Article3.

72. Nookaew I, Papini M, Pornputtapong N, Scalcinati G, Fagerberg L, Uhlen M, Nielsen J: A comprehensive comparison of RNA-seq-based transcriptome analysis from reads to differential gene expression and cross-comparison with microarrays: a case study in Saccharomyces cerevisiae. Nucleic Acids Res 2012, 40(20):10084-10097. 
73. Ferrero $E$, Biswas $P$, Vettoretto $K$, Ferrarini M, Uguccioni M, Piali L, Leone BE, Moser B, Rugarli C, Pardi R: Macrophages exposed to Mycobacterium tuberculosis release chemokines able to recruit selected leucocyte subpopulations: focus on gammadelta cells. Immunology 2003, 108(3):365-374.

74. Langmead B, Trapnell C, Pop M, Salzberg SL: Ultrafast and memoryefficient alignment of short DNA sequences to the human genome. Genome Biol 2009, 10(3):R25.

75. Trapnell C, Pachter L, Salzberg SL: TopHat: discovering splice junctions with RNA-Seq. Bioinformatics 2009, 25(9):1105-1111.

76. R Development Core Team: $R$ : A language and environment for statistical computing. Austria: R Foundation for Statistical Computing Vienna; 2011.

77. Robinson MD, Oshlack A: A scaling normalization method for differential expression analysis of RNA-seq data. Genome Biol 2010, 11(3):R25.

78. Benjamini $Y$, Hochberg $Y$ : Controlling the false discovery rate - a practical and powerful approach to multiple testing. J Roy Stat Soc B Met 1995, 57(1):289-300

79. Wilson $\mathrm{CL}$, Miller CJ: Simpleaffy: a BioConductor package for Affymetrix Quality Control and data analysis. Bioinformatics 2005, 21(18):3683-3685.

80. Wu ZJ, Irizarry RA, Gentleman R, Martinez-Murillo F, Spencer F: A modelbased background adjustment for oligonucleotide expression arrays. J Am Stat Assoc 2004, 99(468):909-917.

81. Hochreiter S, Clevert DA, Obermayer K: A new summarization method for affymetrix probe level data. Bioinformatics 2006, 22(8):943-949.

82. Talloen W, Clevert DA, Hochreiter S, Amaratunga D, Bijnens L, Kass S, Gohlmann HWH: I/NI-calls for the exclusion of non-informative genes: a highly effective filtering tool for microarray data. Bioinformatics 2007, 23(21):2897-2902.

83. Durinck S, Spellman PT, Birney E, Huber W: Mapping identifiers for the integration of genomic datasets with the R/Bioconductor package biomaRt. Nat Protoc 2009, 4(8):1184-1191.

\section{Submit your next manuscript to BioMed Central and take full advantage of:}

- Convenient online submission

- Thorough peer review

- No space constraints or color figure charges

- Immediate publication on acceptance

- Inclusion in PubMed, CAS, Scopus and Google Scholar

- Research which is freely available for redistribution 